\title{
A CONFORMALLY INVARIANT SPHERE THEOREM IN FOUR DIMENSIONS
}

\author{
by Sun-Yung A. CHANG*, Matthew J. GURSKY ${ }^{\star \star}$, and Paul C. YANG ${ }^{\star \star \star}$
}

CONTENTS

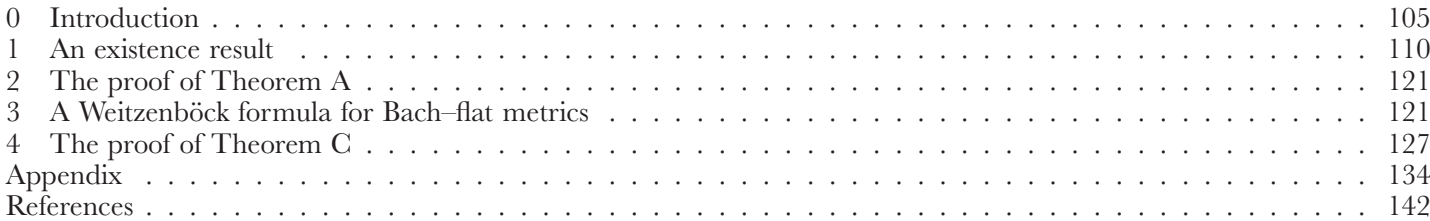

\section{Introduction}

Under what conditions on the curvature can we conclude that a smooth, closed Riemannian manifold is diffeomorphic (or homeomorphic) to the sphere? A result which addresses this question is usually referred to as a sphere theorem, and the literature abounds with examples (see Chapter 11 of [Pe] for a brief survey).

In this paper we concentrate on four dimensions, where Freedman's work is obviously very influential. For example, any curvature condition which implies the vanishing of the de Rham cohomology groups $\mathrm{H}^{1}\left(\mathrm{M}^{4}, \mathbf{R}\right)$ and $\mathrm{H}^{2}\left(\mathrm{M}^{4}, \mathbf{R}\right)$ will, by Freedman's result $([\mathrm{Fr}])$, imply that $\mathbf{M}^{4}$ is covered by a homeomorphism sphere. At the same time, there are some very interesting results which characterize the smooth four-sphere.

An example of particular importance to us is the work of Margerin ([Ma2]), in which he formulated a notion of "weak curvature pinching." To explain this we will need to establish some notation. Given a Riemannian four-manifold $\left(\mathrm{M}^{4}, g\right)$, let Riem denote the curvature tensor, W the Weyl curvature tensor, Ric the Ricci tensor, and R the scalar curvature. The usual decomposition of Riem under the action of $\mathrm{O}(4)$ can be written

$$
\text { Riem }=\mathrm{W}+\frac{1}{2} \mathrm{E} \oslash g+\frac{1}{24} \mathrm{R} g \oslash g,
$$

where $\mathrm{E}=R i c-\frac{1}{4} \mathrm{R} g$ is the trace-free Ricci tensor and $\otimes$ denotes the KulkarniNomizu product. If we let $\mathrm{Z}=\mathrm{W}+\frac{1}{2} \mathrm{E} \otimes g$, then

* Supported by NSF Grant DMS-0070542.

** Supported in part by NSF Grant DMS-9801046 and an Alfred P. Sloan Foundation Research Fellowship.

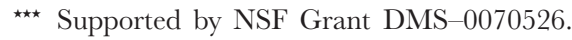




$$
\text { Riem }=\mathrm{Z}+\frac{1}{24} \mathrm{R} g \otimes g .
$$

Note that $\left(\mathrm{M}^{4}, g\right)$ has constant curvature if, and only if, $\mathrm{Z} \equiv 0$. We now define the scale-invariant "weak pinching" quantity

$$
\mathrm{WP} \equiv \frac{|\mathrm{Z}|^{2}}{\mathrm{R}^{2}}=\frac{|\mathrm{W}|^{2}+2|\mathrm{E}|^{2}}{\mathrm{R}^{2}}
$$

where $|Z|^{2}=Z_{i j k l} Z^{i j k l}$ denotes the norm of $Z$ viewed as a $(0,4)$-tensor.

Margerin's main result states that if $\mathrm{R}>0$ and $\mathrm{WP}<\frac{1}{6}$, then $\mathrm{M}^{4}$ is diffeomorphic to either $\mathrm{S}^{4}$ or $\mathbf{R} \mathbf{P}^{4}$. Moreover, this "weak pinching" condition is sharp: The spaces $\left(\mathbf{C P} \mathbf{P}^{2}, g_{\mathrm{FS}}\right)$ and $\left(\mathrm{S}^{3} \times \mathrm{S}^{1}, g_{\text {prod. }}\right)$ both have $\mathrm{R}>0$ and $\mathrm{WP} \equiv \frac{1}{6}$. Indeed, using a holonomy reduction argument, Margerin also proved the converse, in the sense that these manifolds (and any quotients) are characterized by the property that $\mathrm{WP} \equiv \frac{1}{6}$.

Margerin's proof relied on an important tool in the subject of sphere theorems, namely, Hamilton's Ricci flow. In fact, previously Huisken $([\mathrm{Hu}])$ and Margerin ([Mal]) independently had used the Ricci flow to prove a similar pinching result, but with a slightly worse constant. In addition, Hamilton ([Ha]) had used his flow to study four-manifolds with positive curvature operator. As Margerin points out in his introduction, there is no relation between weak pinching and positivity of the curvature operator; indeed, weak pinching even allows for some negative sectional curvature.

On the homeomorphism level, both Margerin's and Hamilton's curvature assumptions already imply that the underlying manifold is covered by a homeomorphism sphere. If $\left(\mathrm{M}^{4}, g\right)$ has positive curvature operator, then the classical Bochner theorem implies that $\mathrm{H}^{1}\left(\mathrm{M}^{4}, \mathbf{R}\right)=\mathrm{H}^{2}\left(\mathrm{M}^{4}, \mathbf{R}\right)=0$. As we observed above, Freedman's work then gives the homeomorphism type of the cover of $\mathbf{M}^{4}$. For manifolds satisfying WP $<\frac{1}{6}$ the vanishing of harmonic forms is less obvious, but does follow from $[\mathrm{Gu}]$.

One drawback to the sphere theorems described above is that they require one to verify a pointwise condition on the curvature. In contrast, consider the (admittedly much simpler) case of surfaces. For example, if the Gauss curvature of the surface $\left(\mathrm{M}^{2}, g\right)$ satisfies $\int \mathrm{K} d \mathrm{~A}>0$, then $\mathrm{M}^{2}$ is diffeomorphic to $\mathrm{S}^{2}$ or $\mathbf{R} \mathbf{P}^{2}$. In addition to this topological classification, the uniformization theorem implies that $\left(\mathrm{M}^{2}, g\right)$ is conformal to a surface of constant curvature, which is then covered isometrically by $\mathrm{S}^{2}$. Therefore, in two dimensions one has a "sphere theorem" which only requires one to check an integral condition on the curvature.

Our goal in this paper is to generalize this situation, by showing that the smooth four-sphere is also characterized by an integral curvature condition. As we shall see, our condition has the additional properties of being sharp and conformally invariant. Although there are different - though equivalent - ways of stating our main result, the simplest version involves the Weyl curvature and the Yamabe invariant: 
Theorem A. - Let $\left(\mathrm{M}^{4}, g\right)$ be a smooth, closed four-manifold for which

(i) the Yamabe invariant $\mathrm{Y}\left(\mathrm{M}^{4}, g\right)>0$, and

(ii) the Weyl curvature satisfies

$(\mathbf{0 . 3})$

$$
\int_{\mathrm{M}^{4}}|\mathrm{~W}|^{2} d v o l<16 \pi^{2} \chi\left(\mathrm{M}^{4}\right) .
$$

Then $\mathrm{M}^{4}$ is diffeomorphic to either $\mathrm{S}^{4}$ or $\mathbf{R} \mathbf{P}^{4}$.

Remarks.

1. Recall that the Yamabe invariant is defined by

$$
\mathrm{Y}\left(\mathrm{M}^{4}, g\right)=\inf _{\tilde{g} \in[g]} \operatorname{vol}(\tilde{g})^{-\frac{1}{2}} \int_{\mathrm{M}^{4}} \mathrm{R}_{\tilde{g}} d v o l \tilde{g},
$$

where $[g]$ denotes the conformal class of $g$. Positivity of the Yamabe invariant implies that $g$ is conformal to a metric of strictly positive scalar curvature.

2. In the statement of Theorem A, the norm of the Weyl tensor is given by $|\mathrm{W}|^{2}=\mathrm{W}_{i j k l} \mathrm{~W}^{i j k l}$; i.e., the usual definition when $\mathrm{W}$ is viewed as a section of $\otimes{ }^{4} \mathrm{~T}^{*} \mathrm{M}^{4}$. However, if one views $\mathrm{W}$ as a section of $\operatorname{End}\left(\Lambda^{2}\left(\mathrm{M}^{4}\right)\right)$, then the convention is $|\mathrm{W}|^{2}=\frac{1}{4} \mathrm{~W}_{i j k l} \mathrm{~W}^{i j k l}$. This can obviously lead to confusion not only when comparing formulas from different sources, but also when the Weyl tensor is interpreted in different ways in the same paper (which will be the case here). To avoid this problem, our convention will be to denote the $(0,4)$-norm using $|\cdot|$, and the $\operatorname{End}\left(\Lambda^{2}\left(\mathrm{M}^{4}\right)\right)$-norm by $\|\cdot\|$, which has the added advantage of emphasizing how we are viewing the tensor in question. We should note that some authors (for example, Margerin) avoid this confusion by just defining an isomorphism between $\otimes^{4} \mathrm{~T}^{*} \mathrm{M}^{4}$ and $\operatorname{End}\left(\Lambda^{2}\left(\mathrm{M}^{4}\right)\right)$ which induces the same norm on both. But in our case, we will adopt the usual identification: if $\mathrm{Z} \in \otimes^{4} \mathrm{~T}^{*} \mathrm{M}^{4}$, then we identify this with $\mathrm{Z} \in \operatorname{End}\left(\Lambda^{2}\left(\mathrm{M}^{4}\right)\right)$ by defining $\mathrm{Z}(\omega)_{i j}=\frac{1}{2} \mathrm{Z}_{i j}{ }^{k l} \omega_{k l}$.

3. That Theorem A relies on an integral curvature condition indicates the possibility one could attempt to formulate a version which imposed much weaker regularity assumptions on the metric. For example, in [CMS], Cheeger, Müller, and Schrader defined a notion of curvature on piecewise flat spaces, which also allowed a generalization of the Chern-Gauss-Bonnet formula.

By appealing to the Chern-Gauss-Bonnet formula, it is possible to replace $(0.3)$ with a condition which does not involve the Euler characteristic. Since

$$
8 \pi^{2} \chi\left(\mathrm{M}^{4}\right)=\int_{\mathrm{M}^{4}}\left(\frac{1}{4}|\mathrm{~W}|^{2}-\frac{1}{2}|\mathrm{E}|^{2}+\frac{1}{24} \mathrm{R}^{2}\right) d v o l,
$$

Theorem $\mathrm{A}$ is equivalent to 
Theorem $\mathbf{A}^{\prime}$. - Let $\left(\mathrm{M}^{4}, g\right)$ be a smooth, closed manifold for which

(i) the Yamabe invariant $\mathrm{Y}\left(\mathrm{M}^{4}, g\right)>0$, and

(ii) the curvature satisfies

$$
\int_{\mathrm{M}^{4}}\left(-\frac{1}{2}|\mathrm{E}|^{2}+\frac{1}{24} \mathrm{R}^{2}-\frac{1}{4}|\mathrm{~W}|^{2}\right) d v o l>0 .
$$

Then $\mathrm{M}^{4}$ is diffeomorphic to either $\mathrm{S}^{4}$ or $\mathbf{R} \mathbf{P}^{4}$.

Formulating the result of Theorem $\mathrm{A}$ in this manner allows us to explain the connection with the work of Margerin. This connection relies on recent work ([CGY1], [CGY2]) in which we established the existence of solutions to a certain fully nonlinear equation in conformal geometry. The relevance of this PDE work to the problem at hand is explained in Section 1. Simply put, the results of [CGY1] and [CGY2] allow us to prove that under the hypotheses of Theorem $\mathrm{A}^{\prime}$, there is a conformal metric for which the integrand in (0.5) is pointwise positive. That is, through a conformal deformation of metric, we are able to pass from positivity in an average sense to pointwise positivity. Now, any metric for which the integrand in (0.5) is positive must satisfy

$$
|\mathrm{W}|^{2}+2|\mathrm{E}|^{2}<\frac{1}{6} \mathrm{R}^{2}
$$

by just rearranging terms. Note that this implies in particular that $\mathrm{R}>0$. Dividing by $\mathrm{R}^{2}$, we conclude that

$$
\mathrm{WP}=\frac{|\mathrm{W}|^{2}+2|\mathrm{E}|^{2}}{\mathrm{R}^{2}}<\frac{1}{6}
$$

The conclusion of the theorem thus follows from Margerin's work.

As we mentioned above, Theorem A is sharp. By this we mean that we can precisely characterize the case of equality:

Theorem B. - Let $\left(\mathrm{M}^{4}, g\right)$ be a smooth, closed manifold which is not diffeomorphic to either $\mathrm{S}^{4}$ or $\mathbf{R P}^{4}$. Assume in addition that

(i) the Tamabe invariant $\mathrm{Y}\left(\mathrm{M}^{4}, g\right)>0$,

(ii) the Weyl curvature satisfies

$$
\int_{\mathrm{M}^{4}}|\mathrm{~W}|^{2} d v o l=16 \pi^{2} \chi\left(\mathrm{M}^{4}\right) .
$$

Then one of the following must be true:

$1\left(\mathrm{M}^{4}, g\right)$ is conformal to $\mathbf{C} \mathbf{P}^{2}$ with the Fubini-Study metric $g_{\mathrm{FS}}$, or

$2\left(\mathrm{M}^{4}, g\right)$ is conformal to a manifold which is isometrically covered by $\mathrm{S}^{3} \times \mathrm{S}^{1}$ endowed with the product metric $g_{\text {prod. }}$. 
The proof of Theorem $\mathrm{B}$ relies on a kind of vanishing result, in a sense which we now explain. Suppose $\left(\mathrm{M}^{4}, g\right)$ satisfies that hypotheses of Theorem B. If there is another metric in a small neighborhood of $g$ for which the $\mathrm{L}^{2}$-norm of the Weyl tensor is smaller; i.e.,

$$
\int_{\mathrm{M}^{4}}|\mathrm{~W}|^{2} d v o l<16 \pi^{2} \chi\left(\mathrm{M}^{4}\right)
$$

then by Theorem A we would conclude that $\mathrm{M}^{4}$ is diffeomorphic to either $\mathrm{S}^{4}$ or $\mathbf{R} \mathbf{P}^{4}$. This, however, contradicts one of the assumptions of Theorem B. Therefore, for every metric in some neighborhood of $g$,

$$
\int_{\mathrm{M}^{4}}|\mathrm{~W}|^{2} d v o l \geq 16 \pi^{2} \chi\left(\mathrm{M}^{4}\right) .
$$

Consequently, $g$ is a critical point (actually, a local minimum) of the Weyl functional $g \mapsto$ $\int|\mathrm{W}|^{2} d v o l$. The gradient of this functional is called the Bach tensor, and we will say that critical metrics are Bach-flat. Note that the conformal invariance of the Weyl tensor implies that Bach-flatness is a conformally invariant property. In fact, the Bach tensor is conformally invariant, [De].

Theorem B is then a corollary of the following classification of Bach-flat metrics:

Theorem C. - Let $\left(\mathrm{M}^{4}, g\right)$ be a smooth, closed manifold which is not diffeomorphic to either $\mathrm{S}^{4}$ or $\mathbf{R} \mathbf{P}^{4}$. Assume in addition that

(i) $\left(\mathrm{M}^{4}, g\right)$ is Bach-flat,

(ii) the Tamabe invariant $\mathrm{Y}\left(\mathrm{M}^{4}, g\right)>0$,

(iii) the Weyl curvature satisfies

$$
\int_{\mathrm{M}^{4}}|\mathrm{~W}|^{2} d v o l=16 \pi^{2} \chi\left(\mathrm{M}^{4}\right) .
$$

Then one of the following must be true:

$1\left(\mathrm{M}^{4}, g\right)$ is conformal to $\mathbf{C} \mathbf{P}^{2}$ with the Fubini-Study metric $g_{\mathrm{FS}}$, or

$2\left(\mathrm{M}^{4}, g\right)$ is conformal to a manifold which is isometrically covered by $\mathrm{S}^{3} \times \mathrm{S}^{1}$ endowed with the product metric $g_{\text {prod. }}$.

In local coordinates, the Bach tensor is given by

$$
\mathrm{B}_{i j}=\nabla^{k} \nabla^{\ell} \mathrm{W}_{k i j \ell}+\frac{1}{2} \mathrm{R}^{k \ell} \mathrm{W}_{k i j \ell} .
$$

Thus, Bach-flatness implies that the Weyl tensor lies in the kernel of a second order differential operator. At the same time, appealing once more to the results of [CGY1] and [CGY2], we can prove that a manifold satisfying the hypotheses of Theorem $\mathrm{C}$ is 
conformal to one for which the integrand in (0.5) is identically zero. In Section 4 we show how these facts, along with a complicated Lagrange-multiplier argument, leads to the classification in Theorem $\mathrm{C}$.

Actually, the proof of Theorem $\mathrm{C}$ is the most technically demanding aspect of the present paper. First, there is a long calculation to derive an integral identity for the covariant derivative of the self-dual and anti-self-dual parts of the Weyl tensor of a Bach-flat metric. In addition to the algebraic difficulties of analyzing the curvature terms which arise in this identity, there are delicate analytic issues. For example, the conformal metric we construct based on the work of [CGY1] and [CGY2] may not be regular on all of $\mathrm{M}^{4}$. Indeed, if it were known to be smooth, then we could appeal to the classification of metrics with $\mathrm{WP} \equiv \frac{1}{6}$ done by Margerin. These regularity problems are the price we pay, so to speak, for passing from integral to pointwise conditions.

We conclude the introduction with a note about the organization of the paper. In Section 1 we develop the necessary PDE material from [CGY1] and [CGY2]. Most of the results are fairly straightforward generalizations of our earlier work. In Section 2 we show how the results of Section 1 and the work of Margerin can be combined to prove Theorem A. Then, in Section 3 we lay the groundwork for the proof of Theorems $\mathrm{B}$ and $\mathrm{C}$ by deriving various identities for the curvature of Bach-flat metrics. In Section 4 we use these identities, along with an existence result from Section 1, to derive a key inequality for a certain polynomial in the curvature. Analyzing this inequality leads us to consider a difficult Lagrange-multiplier problem, whose resolution gives the classification in the statement of Theorem C.

The research for this article was initiated while the second author was a Visiting Professor at Princeton University and the third author was a Visiting Member of the Institute for Advanced Study, and was completed while all three authors were visiting Institut des Hautes Études Scientifiques. The authors wish to acknowledge the support and hospitality of their host institutions.

\section{An existence result}

In this section we prove an existence result in conformal geometry which allows us to pass from the integral conditions of Theorems $\mathrm{A}-\mathrm{C}$ to their pointwise counterparts. As we indicated in the Introduction, this result is based on the work in [CGY1] and [CGY2].

To place this result in its proper context, we begin by introducing some notation. Given a Riemannian four-manifold $\left(\mathrm{M}^{4}, g\right)$, the Weyl-Schouten tensor is defined by

$$
\mathrm{A}=\operatorname{Ric}-\frac{1}{6} \mathrm{R} g
$$


In terms of the Weyl-Schouten tensor, the decomposition (0.1) can be written

$$
\text { Riem }=\mathrm{W}+\frac{1}{2} \mathrm{~A} \otimes g
$$

This splitting of the curvature tensor induces a splitting of the Euler form. To describe this, we introduce the elementary symmetric polynomials $\sigma_{\kappa}: \mathbf{R}^{n} \rightarrow \mathbf{R}$,

$$
\sigma_{\kappa}\left(\lambda_{1}, \ldots, \lambda_{n}\right)=\sum_{i_{1}<\cdots<i_{k}} \lambda_{i_{1}} \cdots \lambda_{i_{\kappa}}
$$

For a section $\mathscr{S}$ of $\operatorname{End}\left(\mathrm{TM}^{4}\right)$ - or, equivalently, a section of $\mathrm{T}^{\star} \mathrm{M}^{4} \otimes \mathrm{TM}^{4}-$ the notation $\sigma_{\kappa}(\mathscr{S})$ means $\sigma_{\kappa}$ applied to the eigenvalues of $\mathscr{S}$. In particular, given a section of the bundle of symmetric two-tensors such as A, by "raising an index" we can cannonically associate a section $g^{-1} \mathrm{~A}$ of $\operatorname{End}\left(\mathrm{TM}^{4}\right)$. At each point of $\mathrm{M}^{4}, g^{-1} \mathrm{~A}$ has 4 real eigenvalues, thus $\sigma_{\kappa}\left(g^{-1} \mathrm{~A}\right)$ is a smooth function on $\mathrm{M}^{4}$. To simplify notation, we denote $\sigma_{\kappa}(\mathrm{A})=\sigma_{\kappa}\left(g^{-1} \mathrm{~A}\right)$.

Returning to the aforementioned splitting of the Euler form, the Chern-GaussBonnet formula (0.4) may be written

$$
8 \pi^{2} \chi\left(\mathrm{M}^{4}\right)=\int \frac{1}{4}|\mathrm{~W}|^{2} d v o l+\int \sigma_{2}(\mathrm{~A}) d v o l .
$$

Note that the conformal invariance of the Weyl tensor implies that the quantity

$$
\int \sigma_{2}(\mathrm{~A}) d v o l
$$

is conformally invariant as well. Using (1.1), assumption (0.5) of Theorem $\mathrm{A}^{\prime}$ can be expressed

$$
\int \sigma_{2}(\mathrm{~A}) d v o l-\int \frac{1}{4}|\mathrm{~W}|^{2} d v o l>0 .
$$

Our goal in this section is to prove the following:

Theorem 1.1. - Let $\left(\mathrm{M}^{4}, g_{0}\right)$ be a smooth, closed Riemannian four-manifold for which

(i) The Tamabe invariant $\mathrm{Y}\left(\mathrm{M}^{4}, g_{0}\right)>0$, and

(ii) The curvature satisfies

$$
\int \sigma_{2}\left(\mathrm{~A}_{g_{0}}\right) d v o l-\int \frac{\alpha}{4}\left|\mathrm{~W}_{g_{0}}\right|^{2} d v o l>0
$$

where $\alpha \geq 0$. Then there is a conformal metric $g_{\alpha}=e^{2 w_{\alpha}} g_{0}$ whose curvature satisfies

$$
\sigma_{2}\left(\mathrm{~A}_{g_{\alpha}}\right)-\frac{\alpha}{4}\left|\mathrm{~W}_{g_{\alpha}}\right|^{2} \equiv \lambda,
$$

where $\lambda$ is a positive constant. 
Theorem 1.1 is a refinement of Corollary B of [CGY2], which for comparison's sake we now state:

Theorem 1.2 (See [CGY2]). - Let $\left(\mathrm{M}^{4}, g_{0}\right)$ be a smooth, closed Riemannian fourmanifold for which

(i) The Yamabe invariant $\mathrm{Y}\left(\mathrm{M}^{4}, g_{0}\right)>0$,

(ii) The curvature satisfies

$$
\int \sigma_{2}\left(\mathrm{~A}_{g_{0}}\right) d v o l_{g_{0}}>0 .
$$

Then there is a conformal metric $g=e^{2 w} g_{0}$ whose curvature satisfies

$$
\sigma_{2}\left(\mathrm{~A}_{g}\right) \equiv \lambda,
$$

where $\lambda$ is a positive constant.

The proof of Theorem 1.2 relies on a crucial preliminary result:

Theorem 1.3 (See [CGY1]). - Let $\left(\mathrm{M}^{4}, g_{0}\right)$ be a smooth, closed Riemannian fourmanifold for which

(i) The Tamabe invariant $\mathrm{Y}\left(\mathrm{M}^{4}, g_{0}\right)>0$,

(ii) The curvature satisfies

$$
\int \sigma_{2}\left(\mathrm{~A}_{g_{0}}\right) d v o l_{g_{0}}>0 .
$$

Then there is a conformal metric $g=e^{2 w} g_{0}$ whose curvature satisfies

$(\mathbf{1 . 8})$

$$
\sigma_{2}\left(\mathrm{~A}_{g}\right)>0
$$

The importance of Theorem 1.3 is that the metric satisfying inequality (1.8) provides an approximate solution to equation (1.6), which can then be deformed to an actual solution. Moreover, (1.8) implies that the path of equations connecting the metric constructed in Theorem 1.3 to the metric constructed in Theorem 1.2 is elliptic.

The proof of Theorem 1.1 will parallel the proofs of Theorems 1.2 and 1.3. The first step is the following analogue of Theorem 1.3:

Theorem 1.4. - Let $\left(\mathrm{M}^{4}, g_{0}\right)$ be a smooth, closed Riemannian four-manifold for which

(i) The Tamabe invariant $\mathrm{Y}\left(\mathrm{M}^{4}, g_{0}\right)>0$,

(ii) The curvature satisfies

$$
\int \sigma_{2}\left(\mathrm{~A}_{g_{0}}\right) d v o l_{g_{0}}-\int \frac{\alpha}{4}\left|\mathrm{~W}_{g_{0}}\right|^{2} d v o l_{g_{0}}>0
$$


where $\alpha \geq 0$. Then there is a conformal metric $g=e^{2 w} g_{0}$ whose curvature satisfies

$(\mathbf{1 . 1 0})$

$$
\sigma_{2}(\mathrm{~A})-\frac{\alpha}{4}|\mathrm{~W}|^{2}>0
$$

Remark. - To simplify notation, we will use subscripts with 0 instead of $g_{0}$, and denote the volume form by $d v_{0}$ instead of $d v o l_{g_{0}}$.

Proof. - Following [CGY1], we consider the following functional F : $\mathrm{W}^{2,2}\left(\mathrm{M}^{4}\right)$ $\rightarrow \mathbf{R}:$

$$
\mathrm{F}[\omega]=\gamma_{1} \mathrm{I}[\omega]+\gamma_{2} \mathrm{II}[\omega]+\gamma_{3} \mathrm{III}[\omega]
$$

where $\gamma_{i}=\gamma_{i}(\mathrm{~L})$ are constants and

$$
\begin{aligned}
\mathrm{I}[\omega] & =\int 4\left|\mathrm{~W}_{0}\right|^{2} \omega d v_{0}-\left(\int\left|\mathrm{W}_{0}\right|^{2} d v_{0}\right) \log f e^{4 \omega} d v_{0}, \\
\mathrm{II}[\omega] & =\int \omega \mathrm{P}_{0} \omega d v_{0}+\int 4 \mathrm{Q}_{0} \omega d v_{0}-\left(\int \mathrm{Q}_{0} d v_{0}\right) \log \int e^{4 \omega} d v_{0}, \\
\mathrm{III}[\omega] & =12\left(\mathrm{Y}[\omega]-\frac{1}{3} \int \Delta_{0} \mathrm{R}_{0} \omega d v_{0}\right), \\
\mathrm{Y}[\omega] & =\int\left(\Delta_{0} \omega+\left|\nabla_{0} \omega\right|^{2}\right)^{2} d v_{0}-\frac{1}{3} \int \mathrm{R}_{0}\left|\nabla_{0} \omega\right|^{2} d v_{0} .
\end{aligned}
$$

Here $\mathrm{P}$ denotes the Paneitz operator:

$$
\mathrm{P}=(\Delta)^{2}+d^{\star}\left(\frac{2}{3} \mathrm{R} g-2 \text { Ric }\right) d,
$$

where $d$ is the exterior derivative, $d^{\star}$ is the adjoint of $d$, and $Q$ is the fourth order curvature invariant:

$$
\mathrm{Q}=\frac{1}{12}\left(-\Delta \mathrm{R}+\frac{1}{4} \mathrm{R}^{2}-3|\mathrm{E}|^{2}\right)
$$

Thus

$$
\mathrm{Q}=\frac{1}{2} \sigma_{2}(\mathrm{~A})+\frac{1}{12}(-\Delta \mathrm{R}) .
$$

As in [CGY2], we need to introduce an additional functional, which depends on the choice of a nowhere--vanishing symmetric $(0,2)$-tensor $\eta$. We then let

$$
\widetilde{\mathrm{I}}[\omega]=\int 4|\eta|_{0}^{2} \omega d v_{0}-\left(\int|\eta|_{0}^{2} d v_{0}\right) \log \int e^{4 \omega} d v_{0}
$$


Now consider the functional

$$
\mathrm{F}[\omega]=\widetilde{\gamma}_{1} \widetilde{I}[\omega]+\gamma_{1} \mathrm{I}[\omega]+\gamma_{2} \mathrm{II}[\omega]+\gamma_{3} \mathrm{III}[\omega],
$$

and define the conformal invariant

$$
\kappa=\tilde{\gamma}_{1} \int|\eta|_{0}^{2} d v_{0}+\gamma_{1} \int\left|\mathbf{W}_{0}\right|^{2} d v_{0}+\gamma_{2} \int \mathbf{Q}_{0} d v_{0} .
$$

Following the work of [CY1], we have the following existence result for extremals of F. To make the paper as self-contained as possible, we will provide a sketch of the proof.

Theorem 1.5 (See [CY1] Theorem 1.1). — Let $\left(\mathrm{M}^{4}, g_{0}\right)$ be a compact Riemannian fourmanifold. If $\gamma_{2}, \gamma_{3}>0$ and $\kappa<\gamma_{2} 8 \pi^{2}$, then $\inf \mathrm{F}[\omega]$ is attained by some function $\omega \in$ $\mathrm{W}^{2,2}\left(\mathrm{M}^{4}\right)$. Moreover, the metric $g=e^{2 \omega} g_{0}$ is smooth (see [CGY3], [UV]) and satisfies

$$
\tilde{\gamma_{1}}|\eta|^{2}+\gamma_{1}|\mathrm{~W}|^{2}+\gamma_{2} \mathrm{Q}-\gamma_{3} \Delta \mathrm{R}=\kappa v o l(g)^{-1} .
$$

Proof. - To see that inf F[ $[\omega]$ is attained under the assumption that $\gamma_{2}, \gamma_{3}>0$ and $\kappa<\gamma_{2} 8 \pi^{2}$, we employ a sharp version of the Moser-Trudinger inequality established by D. Adams [Ad]: there exists a constant $\mathrm{C}=\mathrm{C}\left(\mathrm{M}, g_{0}\right)$ such that for all $\omega \in \mathrm{W}^{2.2}\left(\mathrm{M}, g_{0}\right)$

$$
\log \int e^{4(\omega-\bar{\omega})} d v_{0} \leq \mathrm{C}+\frac{1}{8 \pi^{2}} \int\left(\Delta_{0} \omega\right)^{2} d v_{0},
$$

where $\bar{\omega}=f \omega d v_{0}$.

Define

$$
\mathrm{U}_{0}=\mathrm{U}\left(g_{0}\right)=\tilde{\gamma}_{1}|\eta|_{0}^{2}+\gamma_{1}|\mathrm{~W}|_{0}^{2}+\gamma \mathrm{Q}_{0}-\gamma_{3} \Delta_{0} \mathrm{R}_{0}
$$

then

$$
\int \mathrm{U}_{0} d v_{0}=\kappa
$$

and we can express $\mathrm{F}$ as

$$
\begin{aligned}
\mathrm{F}[\omega]= & -\kappa \log \int e^{4(\omega-\bar{\omega})} d v_{0}+4 \int \mathrm{U}_{0}(\omega-\bar{\omega}) d v_{0} \\
& +\gamma_{2}<\mathrm{P}_{0} \omega, \omega>+12 \gamma_{3} \mathrm{Y}(\omega) .
\end{aligned}
$$

When $\kappa \leq 0$, we have

$$
\mathrm{F}[\omega] \geq 4 \int \mathrm{U}_{0}(\omega-\bar{\omega}) d v_{0}+\gamma_{2}<\mathrm{P}_{0} \omega, \omega>+12 \gamma_{3} \mathrm{Y}(\omega) .
$$


When $\kappa \geq 0$, we have from (1.14) that

$$
\begin{aligned}
\mathrm{F}[\omega] \geq & -\mathrm{C} \kappa-\frac{\kappa}{8 \pi^{2}} \int\left(\Delta_{0} \omega\right)^{2} d v_{0} \\
& +4 \int \mathrm{U}_{0}(\omega-\bar{\omega}) d v_{0}+\gamma_{2}<\mathrm{P}_{0} \omega, \omega>+12 \gamma_{3} \mathrm{Y}(\omega)
\end{aligned}
$$

Thus, for a minimizing sequence $\left\{\omega_{l}\right\}, \underline{\lim } \mathrm{F}\left[\omega_{l}\right] \leq \mathrm{F}[0]=0$. From the estimates above we conclude that for $l$ large, $\epsilon$ small,

$$
\begin{aligned}
\epsilon \geq \mathrm{F}\left[\omega_{l}\right] \geq & -\mathrm{C} \tilde{\kappa}+\left(-\frac{\tilde{\kappa}}{8 \pi^{2}}+\gamma_{2}+12 \gamma_{3}\right) \int\left(\Delta_{0} \omega_{l}\right)^{2} \\
& +12 \gamma_{3} \int\left|\nabla_{0} \omega_{l}\right|^{4}+4 \int \mathrm{U}_{0}\left(\omega_{l}-\bar{\omega}_{l}\right) \\
& +\left(\frac{2}{3} \gamma_{2}-4 \gamma_{3}\right) \int \mathrm{R}_{0}\left|\nabla_{0} \omega_{l}\right|^{2}-2 \gamma_{2} \int \operatorname{Ric}_{0}\left(\nabla_{0} \omega_{l}, \nabla_{0} \omega_{l}\right) \\
& +24 \gamma_{3} \int\left(\Delta_{0} \omega_{l}\right)\left|\nabla_{0} \omega_{l}\right|^{2}
\end{aligned}
$$

where $\tilde{\kappa}=\max (\kappa, 0)$. It follows that if $\kappa \leq \gamma_{2} 8 \pi^{2}$, and $\gamma_{2}>0, \gamma_{3}>0$, one concludes that there exists a constant $\mathrm{C}\left(g_{0}\right)$ so that

$$
\int\left(\Delta_{0} \omega_{l}\right)^{2}+\left|\nabla_{0} \omega_{l}\right|^{4} \leq \mathrm{C}\left(g_{0}\right)
$$

Since the functional $\mathrm{F}$ is scale-invariant, we may assume without loss of generality that $f \omega_{l} d v_{0}=0$. It follows from the Poincare inequality and (1.15) that $\left\|\omega_{l}\right\|_{\mathrm{L}^{2}}$ is uniformly bounded. Therefore, $\left\|\omega_{l}\right\|_{2,2}$ is bounded and a subsequence will converge weakly in $\mathrm{W}^{2,2}$ to some $\omega \in \mathrm{W}^{2,2}\left(\mathrm{M}, g_{0}\right)$ with $\mathrm{F}[\omega]=\inf _{\omega \in \mathrm{W}^{2,2}} \mathrm{~F}[\omega]$.

Finally, a straightforward computation (see [BO]) shows that the extremal metric $g=e^{2 \omega} g_{0}$ satisfies the Euler equation (1.13).

Next, let $\delta \in(0,1]$ and choose

$$
\begin{aligned}
& \tilde{\gamma}_{1}=\left(-\frac{1}{2}\right) \frac{\int \sigma_{2}\left(\mathrm{~A}_{0}\right) d v_{0}-\frac{\alpha}{4} \int\left|\mathrm{W}_{0}\right|^{2} d v_{0}}{\int|\eta|_{0}^{2} d v_{0}}<0, \\
& \gamma_{1}=-\frac{\alpha}{8}, \\
& \gamma_{2}=1, \\
& \gamma_{3}=\frac{1}{24}(3 \delta-2) .
\end{aligned}
$$


With these values of $\left(\widetilde{\gamma}_{1}, \gamma_{1}, \gamma_{2}, \gamma_{3}\right)$, the conformal invariant $\kappa$ defined in (1.12) is equal to zero. Thus, as long as $\gamma_{3}>0$ (i.e., $\delta>\frac{2}{3}$ ) the hypotheses of Theorem 1.5 hold. In particular, if $\delta=1$ there exists an extremal metric $g_{1}=e^{2 \omega} g_{0}$, satisfying (1.13), which we rewrite using (1.11):

$$
\sigma_{2}(\mathrm{~A})-\frac{\alpha}{4}|\mathrm{~W}|^{2}=\frac{1}{4} \Delta \mathrm{R}-2 \tilde{\gamma}_{1}|\eta|^{2} .
$$

Using the minimum principle of [Gu, Lemma 1.2], this implies that the scalar curvature of $g_{1}$ is strictly positive.

For general $\delta \in(0,1]$, the Euler equation (1.13) can be written

$$
(\star \star)_{\delta} \quad \sigma_{2}(\mathrm{~A})-\frac{\alpha}{4}|\mathrm{~W}|^{2}=\frac{\delta}{4} \Delta \mathrm{R}-2 \widetilde{\gamma}_{1}|\eta|^{2} .
$$

Compare this with equation $(\star)_{\delta}$ of $[\mathrm{CGY} 1]$ :

$$
\sigma_{2}(\mathrm{~A})=\frac{\delta}{4} \Delta \mathrm{R}-2 \gamma_{1}|\eta|^{2}
$$

Note that the only discrepancy between $(\star \star)_{\delta}$ and $(\star)_{\delta}$ is the presence of the Weyl term on the LHS of $(\star)_{\delta}$. The key point is that this term has the same sign as the term involving $\eta$ and scales in exactly the same way. That is, if $g=e^{2 w} g_{0}$ is a conformal metric, then

$$
\begin{gathered}
\left|\mathrm{W}_{g}\right|_{g}^{2}=e^{-4 w}\left|\mathrm{~W}_{0}\right|_{0}^{2}, \\
|\eta|_{g}^{2}=e^{-4 w}|\eta|_{0}^{2} .
\end{gathered}
$$

Consequently, the subsequent arguments of Sections 2-6 of [CGY1] can be carried out with only trivial modifications, as we now describe.

Fixing $\delta_{0}>0$, let

$$
\mathscr{S}=\left\{\begin{array}{l|l}
\delta \in\left[\delta_{0}, 1\right] & \begin{array}{l}
(\star \star)_{\delta} \text { admits a solution with } \\
\text { positive scalar cuvature }
\end{array}
\end{array}\right\} .
$$

As we saw above, $1 \in \mathscr{S}$; thus $\mathscr{S}$ is non-empty. To verify that $\mathscr{S}$ is open we compute the linearization of $(\star \star)_{\delta}$, exactly as in Proposition 4.1 of [CGY2]. Again, the only relevant properties of the Weyl term and $\eta$-term in $(\star \star)_{\delta}$ are their scaling properties (which are the same) and their sign (ditto). Next, the estimates of Section 3 in [CGY1] can be be used to show that $\mathscr{S}$ is closed. Consequently, for each $\delta>0$, there is a conformal metric $g=g_{\delta}=e^{2 \omega_{\delta}} g_{0}$ of positive scalar curvature satisfying $(\star \star)_{\delta}$.

In Sections 4-6 of [CGY2] we obtain the following a priori estimate for solutions of $(\star)_{\delta}$ : For fixed $p<5$, there is a constant $\mathrm{C}=\mathrm{C}(p)$ such that

$$
\left\|\omega_{\delta}\right\|_{2, p} \leq \mathrm{C} .
$$


In particular, $\mathrm{C}$ is independent of $\delta$. The same estimate holds for solutions of $(\star \star)_{\delta}$, for the reasons explained above.

In Section 7 of [CGY1] the Yamabe flow is used to show that one can perturb solutions of $(\star)_{\delta}$ to find metrics with $\sigma_{2}(\mathrm{~A})>0$. An analogous result is true for solutions of $(\star \star)_{\delta}$.

Theorem 1.6 (See Theorem 7.1 of [CGY1]). — Let $g=e^{2 \omega} g_{0}$ be a solution of $(\star \star)_{\delta}$ with positive scalar curvature, normalized so that $\int \omega d v_{0}=0$. If $\delta>0$ is sufficiently small, then there is a smooth conformal metric $h=e^{2 v} g$ with

$$
\sigma_{2}\left(\mathrm{~A}_{h}\right)-\frac{\alpha}{4}\left|\mathrm{~W}_{h}\right|^{2}>0 .
$$

Proof. - The proof of Theorem 1.6, like the proof of its counterpart Theorem 7.1 of [CGY1], is based on careful estimates of solutions to the Yamabe flow:

$$
\begin{gathered}
\frac{\partial h}{\partial t}=-\frac{1}{3} \mathrm{R} h, \\
h(0, \cdot)=g=e^{2 \omega} g_{0} .
\end{gathered}
$$

Using the estimate (1.17), we show that there is a time $\mathrm{T}_{0}$, which only depends on the background metric $g_{0}$, such that the metric $h=h\left(\mathrm{~T}_{0}, \cdot\right)$ satisfies (1.18). Although the arguments are essentially the same, there are some necessary modifications of the proof of Theorem 7.1 which require explanation.

First, Propositions 7.2 and 7.4, and Lemmas 7.3 and 7.5 can all be copied without change. In the statement of Proposition 7.8 we need to make an obvious change: instead of defining $f=\sigma_{2}(\mathrm{~A})+2 \gamma_{1}|\eta|^{2}$, we define $\tilde{f}=\sigma_{2}(\mathrm{~A})-\frac{\alpha}{4}|\mathrm{~W}|^{2}+2 \tilde{\gamma}_{1}|\eta|^{2}$. The conclusion of Proposition 7.8 then holds with $f$ replaced by $\tilde{f}$.

In fact, by substituting $f$ with $\tilde{f}$, the proof of the next proposition (7.12) is also valid. Therefore, by following the remaining arguments of Section 7 we arrive at the following inequality: For fixed $s \in(4,5)$ and $t \leq \mathrm{T}_{1}\left(g_{0}\right)$,

$$
\sigma_{2}(\mathrm{~A})-\frac{\alpha}{4}|\mathrm{~W}|^{2} \geq-2 \widetilde{\gamma}_{1}|\eta|^{2}-\mathrm{C}_{3} t^{1-\frac{4}{s}}-\mathrm{C}_{3} \delta^{\frac{1}{2}} t^{\left(-1+\frac{2}{s}\right)},
$$

where $\mathrm{C}_{3}=\mathrm{C}_{3}\left(g_{0}\right)$. Since $s>4$ and $-2 \widetilde{\gamma}_{1}|\eta|^{2} \geq \mathrm{C}\left(g_{0}\right)>0$, it follows there is a constant $\mathrm{C}_{4}=\mathrm{C}_{4}\left(g_{0}\right)>0$ so that for $t \leq \mathrm{T}_{0}=\mathrm{T}_{0}\left(g_{0}\right)$,

$$
\sigma_{2}(\mathrm{~A})-\frac{\alpha}{4}|\mathrm{~W}|^{2} \geq \frac{3}{4} \mathrm{C}_{4}-\mathrm{C}_{3} \delta^{\frac{1}{2}} t^{-\left(1+\frac{2}{s}\right)} .
$$

Therefore, if $h=\left(t_{0}, \cdot\right)$, then for $\delta<\delta_{0}\left(g_{0}\right),(1.19)$ implies

$$
\sigma_{2}\left(\mathrm{~A}_{h}\right)-\frac{\alpha}{4}\left|\mathrm{~W}_{h}\right|^{2} \geq \frac{1}{2} \mathrm{C}_{4}>0 .
$$

This completes the proof of Theorem 1.6, and consequently Theorem 1.4. 
Once the existence of a metric satisfying (1.18) is established, to complete the proof of Theorem 1.1 we need to show that the techniques of [CGY2] can be applied to construct a solution of (1.4). This is actually a two step process: First, we need to establish a priori bounds for solutions of

$$
\sigma_{2}\left(\mathrm{~A}_{g}\right)-\frac{\alpha}{4}\left|\mathrm{~W}_{g}\right|^{2}=f>0
$$

The second step is to apply a degree-theoretic argument showing that a metric satisfying (1.18) can be deformed to a metric satisfying (1.4). Of course, such an argument relies on the estimates established in the first step.

Proposition 1.7 (See Main Theorem of [CGY2]). — Let $g=e^{2 w} g_{0}$ be a solution of (1.20) with positive scalar curvature, and assume $\left(\mathrm{M}^{4}, g_{0}\right)$ is not conformally equivalent to the round sphere. Then there is a constant $\mathrm{G}=\mathrm{G}\left(g_{0},\|f\|_{\mathrm{C}^{2}}\right)$ such that

$$
\max _{\mathrm{M}^{4}}\left\{e^{w}+\left|\nabla_{0} w\right|\right\} \leq \mathrm{C} .
$$

Now the estimate of [CGY2] applies to equations of the form

$$
\sigma_{2}\left(\mathrm{~A}_{g}\right)=f>0
$$

whereas (1.20) includes the Weyl term. However, the argument of [CGY2] can easily be modified to cover this case, as we now explain.

As in [CGY2] we argue by contradicition: assuming the theorem is false, then there is a sequence of solutions $\left\{w_{\kappa}\right\}$ of (1.20) (with $f$ fixed) such that

$$
\max _{M}\left[\left|\nabla_{0} w_{\kappa}\right|+e^{w_{\kappa}}\right] \rightarrow \infty \quad \text { as } \quad \kappa \rightarrow \infty .
$$

We then apply the blow-up argument described pages 155-156 of [CGY2]. To begin, assume that $\mathrm{P}_{\kappa} \in \mathbf{M}^{4}$ is a point at which $\left(\left|\nabla_{0} w_{\kappa}\right|+e^{w_{k}}\right)$ attains its maximum. By choosing normal coordinates $\left\{\Phi_{\kappa}\right\}$ centered at $\mathrm{P}_{\kappa}$, we may identify the coordinate neighborhood of $\mathrm{P}_{\kappa}$ in $\mathrm{M}^{4}$ with the unit ball $\mathrm{B}(1) \subset \mathbf{R}^{4}$ such that $\Phi_{\kappa}\left(\mathrm{P}_{\kappa}\right)=0$. Given $\varepsilon>0$, we define the dilations $\mathrm{T}_{\varepsilon}: \mathbf{R}^{4} \rightarrow \mathbf{R}^{4}$ by $x \mapsto \varepsilon x$, and consider the sequence $w_{\kappa, \varepsilon}=\mathrm{T}_{\varepsilon}^{\star} w_{\kappa}+\log \varepsilon$. Note that

$$
\left|\nabla_{0} w_{\kappa, \varepsilon}\right|+e^{w, \varepsilon}=\varepsilon\left(\left|\nabla_{0} w_{\kappa}\right|+e^{w_{\kappa}}\right) \circ \mathrm{T}_{\varepsilon} .
$$

Thus, for each $\kappa$ we can choose $\varepsilon_{\kappa}$ so that

$$
\left|\nabla_{0}\left(w_{\kappa, \varepsilon_{\kappa}}\right)\right|+\left.e^{w_{\kappa, \varepsilon_{\kappa}}}\right|_{x=0}=1 .
$$

Note that $w_{\kappa, \varepsilon_{\kappa}}$ is defined in $\mathrm{B}_{\frac{1}{\varepsilon_{\kappa}}}(0)$, and

$$
\left|\nabla_{0}\left(w_{\kappa, \varepsilon_{\kappa}}\right)\right|+e^{w_{\kappa}, \varepsilon_{\kappa}} \leqslant 1 \quad \text { on } \quad \mathrm{B}_{\frac{1}{\varepsilon_{\kappa}}}(0) .
$$


To simplify notation, let us denote $w_{\kappa, \varepsilon_{\kappa}}$ by $w_{\kappa}$. Since from now on we view $\left\{w_{\kappa}\right\}$ as a sequence defined on dilated balls in $\mathbf{R}^{4}$, there will be no danger of confusing the renormalized sequence with the original sequence. Note that $g_{\kappa}^{\star} \equiv e^{2 w_{\kappa}} \mathrm{T}_{\varepsilon_{\kappa}}^{\star} g_{0} \equiv e^{2 w_{\kappa}} g_{0}^{\kappa}$ satisfies

$$
\sigma_{2}\left(\mathrm{~A}_{g_{\kappa}}\right)-\frac{\alpha}{4}\left|\mathrm{~W}_{g_{\kappa}^{\star}}\right|^{2}=f \circ \mathrm{T}_{\varepsilon_{k}} .
$$

Furthermore, $g_{0}^{\kappa}=\mathrm{T}_{\varepsilon_{\kappa}}^{\star} g_{0} \rightarrow d s^{2}$; where $d s^{2}$ is the Euclidean metric on $\mathbf{R}^{4}$, in $\mathrm{C}^{2, \beta}$ on compact sets.

As in [CGY2], we now have to consider two possibilities, depending on the behavior of the exponential term $e^{w_{k}(0)}$. However, from here on the argument is identical in its details with that of [CGY2]. The main point is that the conformal invariance of the Weyl curvature implies that the two possible limit metrics arising from the sequence $g_{\kappa}^{\star}$ satisfy the same equations as they do in [GGY2]; that is, the Weyl term in (1.22) converges to zero because Euclidean space is conformally flat. In particular, Corollary 1.3 in [CGY3] applies to equations such as (1.20), so the estimates needed to construct the limiting metric are the same.

To summarize: after applying the same blow-up argument to our sequence, we end up with the same limiting equations on $\mathbf{R}^{4}$ (see Corollary 1.4 in [CGY2]). The rest of the proof carries through exactly as in [CGY2], and we conclude that the manifold $\left(\mathrm{M}^{4}, g\right)$ must be conformally equivalent to the round 4-sphere. Since this contradicts our assumption, the estimate (1.21) must hold.

The local estimate of [CGY2, Cor 1.3] also applies, and consequently we have a bound

$$
\left\|\nabla^{2} w\right\|_{\infty} \leq \mathrm{G} .
$$

Next, we use a degree theoretic argument to prove the existence of a solution of (1.4). The following proposition is a fairly straightforward modification of Corollary B in [GGY2].

Proposition 1.8. - Assume that $\left(\mathrm{M}^{4}, g_{0}\right)$ satisfies conditions (i) and (ii) of Theorem 1.4. Then given any positive (smooth) function $f>0$, there exists a solution $g=e^{2 w} g_{0}$ of (1.20). In particular, this is true if $f \equiv \lambda$ for a constant $\lambda>0$.

Proof. - We first apply Theorem 1.4 to assert the existence of a conformal metric $g=e^{2 w} g_{0}$ for which the equation (1.20) holds for some positive function $f$. Since by assumption $\left(\mathrm{M}^{4}, g_{0}\right)$ is not conformally equivalent to the standard 4-sphere, the a priori estimates (1.22) and (1.23) hold. In particular, given a smooth function $h$, there is a constant $c$ independent of $t$ so that all solutions $g=e^{2 w} g_{0}$ of the equation

$$
\sigma_{2}\left(\mathrm{~A}_{g}\right)-\frac{\alpha}{4}\left|\mathrm{~W}_{g}\right|^{2}=t f+(1-t) h
$$


with $\mathrm{R}=\mathrm{R}_{g}>0$ satisfy the bounds

$$
\|w\|_{4, \alpha} \leqslant c, \quad \mathrm{~S}_{i j}(g) \xi_{i} \xi_{j} \geqslant \frac{1}{c}|\xi|^{2},
$$

where $\mathrm{S}=-\operatorname{Ric}+\frac{1}{2} \mathrm{R} g$ (see [CGY1], Lemma 1.2). Let $\mathrm{O}_{c}$ be the set

$$
\begin{aligned}
\mathrm{O}_{c}= & \left\{w \in \mathrm{C}^{4, \alpha}:(1.24) \text { holds }\right\} \\
& \cap\left\{w \in \mathrm{C}^{4, \alpha} \mid\left(\sigma_{2}\left(\mathrm{~A}_{g_{w}}\right)-\frac{\alpha}{4}\left|\mathrm{~W}_{g_{w}}\right|^{2}\right)>0 ; \mathrm{R}_{g_{w}}>0\right\} .
\end{aligned}
$$

We denote the degree of the equation $\left(\Sigma_{t}\right)$ by $\operatorname{deg}\left(\Sigma_{t}, \mathrm{O}_{c}, 0\right)$. The degree theory of [Li] implies that

$$
\operatorname{deg}\left(\Sigma_{0}, \mathrm{O}_{c}, 0\right)=\operatorname{deg}\left(\Sigma_{1}, \mathrm{O}_{c}, 0\right) .
$$

We need to do a calculation verifying that for $t=1$ the degree of the equation is non-zero. In order to do this, we deform the equation to one whose degree is easy to determine. First, it is useful to re-write equation (1.20) in a suggestive form. Suppose $g=e^{2 w} g_{0}$ and denote

$$
\mathbf{M}_{i j}(w)=2 \mathrm{~S}_{i j}^{0}+2 \nabla_{i}^{0} \nabla_{j}^{0} w-2 \Delta_{0} w g_{i j}^{0}-2 \nabla_{i}^{0} w \nabla_{j}^{0} w .
$$

Then, after some computation, the equation (1.20) may be written in the form

$$
\begin{aligned}
-\nabla_{i}^{0}\left\{\mathrm{M}_{i j}(w) \nabla_{j}^{0} w\right\}+\left(\sigma_{2}\left(\mathrm{~A}_{g_{0}}\right)-\frac{\alpha}{4}\left|\mathrm{~W}_{g_{0}}\right|^{2}\right) & =\left(\sigma_{2}\left(\mathrm{~A}_{g}\right)-\frac{\alpha}{4}\left|\mathrm{~W}_{g}\right|^{2}\right) e^{4 w} \\
& =f e^{4 w} .
\end{aligned}
$$

It is important to note the identity

$$
\mathrm{M}_{i j}(w)=\mathrm{S}_{i j}+\mathrm{S}_{i j}^{0}+\left|\nabla_{0} w\right|^{2} g_{i j}^{0}
$$

so that it is clear that when both $\left(\sigma_{2}\left(\mathrm{~A}_{g}\right)-\frac{\alpha}{4}\left|\mathbf{W}_{g}\right|^{2}\right)>0, \mathbf{R}_{g}>0$ and $\left(\sigma_{2}\left(\mathrm{~A}_{g_{0}}-\right.\right.$ $\left.\frac{\alpha}{4}\left|\mathbf{W}_{g_{0}}\right|^{2}\right)>0, \mathbf{R}_{g_{0}}>0$, then $\mathbf{M}_{i j}$ is positive definite.

It is also convenient to re-formulate equation (1.20), on account of the conformal covariance property, using the solution metric $g$ of the equation as the background metric:

$$
-\nabla_{i}\left\{\mathbf{M}_{i j}(v) \nabla_{j} v\right\}+f=f e^{4 v},
$$

so that $v=0$ is a solution to this equation satisfying $\mathbf{R}>0$.

We now use the following deformation:

$$
-\nabla_{i}\left\{\mathrm{M}_{i j}(v) \nabla_{j} v\right\}+f=\left(\sigma_{2}\left(\mathrm{~A}_{g_{v}}\right)-\frac{\alpha}{4}\left|\mathrm{~W}_{g_{v}}\right|^{2}\right) e^{4 v}=(1-t) f \int e^{4 v}+t f e^{4 v}
$$


where $\int e^{4 v}=\int e^{4 v} d v o l_{g}$. We label this equation by $\Gamma_{t}$. Note that when $t=1$, we recover the equation (1.28). The proof of the proposition now follows line by line the proof of Corollary B in [CGY2]. More precisely, after establishing a priori estimates for solutions of $\Gamma_{t}$, we find that the degree is well defined. A calculation of the linearized equation, together with the homotopy invariance of the degree implies that $\operatorname{deg}\left(\Gamma_{1}, \mathrm{O}_{c}, 0\right)=\operatorname{deg}\left(\Gamma_{0}, \mathrm{O}_{c}, 0\right)=-1$. It follows that a solution of $(1.20)$ exists.

Applying Proposition 1.8, we complete the proof of Theorem 1.1.

\section{The proof of Theorem $A$}

Based on the results of Section 1, we can now give a detailed proof of Theorem A.

As we saw above, the assumption (0.3) is equivalent to the inequality (1.2):

$$
\int_{\mathrm{M}^{4}} \sigma_{2}(\mathrm{~A}) d v o l-\int_{\mathrm{M}^{4}} \frac{1}{4}|\mathrm{~W}|^{2} d v o l>0 .
$$

Taking $\alpha=1$ in Theorem 1.1, it follows that there is a conformal metric satisfying

$$
\sigma_{2}(\mathrm{~A})-\frac{1}{4}|\mathrm{~W}|^{2} \equiv \lambda>0 \text {. }
$$

Strictly speaking, at this stage all we really need is the conclusion of Theorem 1.4 - that is, we just need to know that the quantity in (2.1) is positive, not necessarily constant.

In any case, rewriting $\sigma_{2}(\mathrm{~A})$ in terms of the trace-free Ricci tensor $\mathrm{E}=$ Ric $\frac{1}{4} \mathrm{R} g$ and the scalar curvature we conclude

$$
-\frac{1}{2}|\mathrm{E}|^{2}+\frac{1}{24} \mathrm{R}^{2}-\frac{1}{4}|\mathrm{~W}|^{2}>0 .
$$

Rearranging terms, this implies

$$
\frac{|\mathrm{W}|^{2}+2|\mathrm{E}|^{2}}{\mathrm{R}^{2}}<\frac{1}{6} .
$$

By the weak-pinching result of Margerin [Ma2], $\mathrm{M}^{4}$ is diffeomorphic to $\mathrm{S}^{4}$ or $\mathbf{R} \mathbf{P}^{4}$.

\section{A Weitzenböck formula for Bach-flat metrics}

In preparation for the proof of Theorem $\mathrm{C}$, in this section we derive various curvature identities. The first such result is an inequality for metrics satisfying (1.4). 
Lemma 3.1. - Suppose $\left(\mathrm{M}^{4}, g\right)$ satisfies (1.4):

$$
\sigma_{2}(\mathrm{~A})-\frac{\alpha}{4}|\mathrm{~W}|^{2}=\lambda
$$

where $\alpha \geq 0$ and $\lambda \geq 0$ are constants. Then

$$
\frac{3}{2} \alpha|\nabla \mathrm{W}|^{2}+3\left(|\nabla \mathrm{E}|^{2}-\frac{1}{12}|\nabla \mathrm{R}|^{2}\right) \geq 0 .
$$

Proof. - Define the tensor

$$
\mathrm{V}=\sqrt{\alpha} \mathrm{W}+\frac{1}{2} \mathrm{E} \oslash g
$$

where $($ is the Kulkarni-Nomizu product. Then

$$
\begin{aligned}
|\mathrm{V}|^{2} & =\alpha|\mathrm{W}|^{2}+2|\mathrm{E}|^{2} \\
|\nabla \mathrm{V}|^{2} & =\alpha|\nabla \mathrm{W}|^{2}+2|\nabla \mathrm{E}|^{2} .
\end{aligned}
$$

As a consequence of (3.3), inequality (3.1) is equivalent to

$$
|\nabla \mathrm{V}|^{2} \geq \frac{1}{6}|\nabla \mathrm{R}|^{2}
$$

To verify (3.4) note that (1.4) and (3.2) imply

$$
\frac{1}{6} \mathrm{R}^{2}=|\mathrm{V}|^{2}+4 \lambda
$$

Differentiating,

$$
\frac{1}{3} \mathrm{R} \nabla \mathrm{R}=\nabla|\mathrm{V}|^{2}=2|\mathrm{~V}| \nabla|\mathrm{V}| .
$$

Taking the inner product of both sides with $\mathrm{R}^{-1} \nabla \mathrm{R}$ gives

$$
\frac{1}{3}|\nabla \mathrm{R}|^{2}=2|\mathrm{~V}| g\left(\nabla|\mathrm{V}|, \frac{\nabla \mathrm{R}}{\mathrm{R}}\right) \leq|\mathrm{V}|^{2} \frac{|\nabla \mathrm{R}|^{2}}{\mathrm{R}^{2}}+|\nabla| \mathrm{V}||^{2} .
$$

By Kato's inequality, $|\nabla| \mathrm{V}||^{2} \leq|\nabla \mathrm{V}|^{2}$, and thus

$$
\frac{1}{3}|\nabla \mathrm{R}|^{2} \leq|\mathrm{V}|^{2} \frac{|\nabla \mathrm{R}|^{2}}{\mathrm{R}^{2}}+|\nabla \mathrm{V}|^{2}
$$

Substituting (3.5) into (3.6) gives

$$
\frac{1}{3}|\nabla \mathrm{R}|^{2} \leq\left(\frac{1}{6} \mathrm{R}^{2}-4 \lambda\right) \frac{|\nabla \mathrm{R}|^{2}}{\mathrm{R}^{2}}+|\nabla \mathrm{V}|^{2} \leq \frac{1}{6}|\nabla \mathrm{R}|^{2}+|\nabla \mathrm{V}|^{2},
$$

because $\lambda \geq 0$. This establishes (3.4), and consequently (3.1). 
We now recall two important identities for Bach-flat metrics. The first may be found in [GGY1]:

Proposition 3.2 (See [GGY1], Lemma 5.4). - If $\left(\mathrm{M}^{4}, g\right)$ is Bach-flat, then

$$
0=\int_{\mathrm{M}^{4}}\left\{3\left(|\nabla \mathrm{E}|^{2}-\frac{1}{12}|\nabla \mathrm{R}|^{2}\right)+6 t r \mathrm{E}^{3}+\mathrm{R}|\mathrm{E}|^{2}-6 \mathrm{~W}_{i j k \ell} \mathrm{E}_{i k} \mathrm{E}_{j \ell}\right\} d v o l,
$$

where $t \mathrm{r} \mathrm{E}^{3}=\mathrm{E}_{i j} \mathrm{E}_{i k} \mathrm{E}_{j k}$.

Proof. - Just combine identities (5.5) and (5.10) of [GGY1].

The second identity is a consequence of Stokes' Theorem, the Bianchi identities, and the definition of the Bach tensor in (0.7).

Proposition 3.3. - If $\left(\mathrm{M}^{4}, g\right)$ is Bach flat, then

$$
\int_{\mathrm{M}^{+}}|\nabla \mathrm{W}|^{2} d v o l=\int\left\{72 \operatorname{det} \mathrm{W}^{+}+72 \operatorname{det} \mathrm{W}^{-}-\frac{1}{2} \mathrm{R}|\mathrm{W}|^{2}+2 \mathrm{~W}_{i j k \ell} \mathrm{E}_{i k} \mathrm{E}_{j \ell}\right\} .
$$

Proof. - In [De], Derdzinski proved a similar formula for metrics with harmonic Weyl tensor. Our identity differs from his in only one respect; namely, we replace harmonicity of the Weyl tensor (a first order condition) with Bach flatness (a second order condition). To simplify the calculations and harmonize our notation with [De], we compute with respect to a local (normal) frame field. Using this convention, we write

$$
\int\left|\nabla \mathrm{W}^{+}\right|^{2}=\int \nabla_{m} \mathrm{~W}_{i j k \ell}^{+} \nabla_{m} \mathrm{~W}_{i j k \ell}^{+} \text {. }
$$

Since the splitting $\Lambda^{2}=\Lambda_{+}^{2} \oplus \Lambda_{-}^{2}$ is parallel with respect to the Riemannian connection,

$$
\nabla \mathrm{W}=\nabla \mathrm{W}^{+}+\nabla \mathrm{W}^{-} .
$$

Therefore,

$$
\int\left|\nabla \mathrm{W}^{+}\right|^{2}=\int \nabla_{m} \mathrm{~W}_{i j k \ell} \nabla_{m} \mathrm{~W}_{i j k \ell}^{+} .
$$

Using the decomposition (1.0), the second Bianchi identity can be written

$$
\begin{aligned}
0= & \nabla_{m} \mathrm{~W}_{i j k \ell}+\nabla_{i} \mathrm{~W}_{j m k \ell}+\nabla_{j} \mathrm{~W}_{m i k \ell} \\
& +\frac{1}{2}\left[g_{i k}(d \mathrm{~A})_{m j \ell}-g_{i \ell}(d \mathrm{~A})_{m j k}\right. \\
& -g_{j k}(d \mathrm{~A})_{m i \ell}+g_{j \ell}(d \mathrm{~A})_{m i k}-g_{k m}(d \mathrm{~A})_{i j \ell} \\
& \left.+g_{m \ell}(d \mathrm{~A})_{i j k}\right],
\end{aligned}
$$


where

$$
(d \mathrm{~A})_{i j k}=\nabla_{i} \mathrm{~A}_{j k}-\nabla_{j} \mathrm{~A}_{i k}
$$

Contracting (3.12) we get the identity

$$
(\delta \mathrm{W})_{i j \ell} \equiv \nabla_{m} \mathrm{~W}_{i j m \ell}=\frac{1}{2}(d \mathrm{~A})_{i j \ell},
$$

where $\delta$ denotes the divergence. Subtituting (3.12) into (3.11) we get

$$
\begin{aligned}
\int\left|\nabla \mathrm{W}^{+}\right|^{2}= & \int \nabla_{m} \mathrm{~W}_{j k \ell}^{+}\left\{-\nabla_{i} \mathrm{~W}_{j m k \ell}\right. \\
& \left.-\nabla_{j} \mathrm{~W}_{m i k \ell}+\frac{1}{2}\left[g_{k m}(d \mathrm{~A})_{i j \ell}-g_{m \ell}(d \mathrm{~A})_{i j k}\right]\right\},
\end{aligned}
$$

because all other terms vanish due to the symmetries of the Weyl tensor. Re-indexing and combining like terms, we find

$$
\int\left|\nabla \mathrm{W}^{+}\right|^{2}=\int-2 \nabla_{m} \mathrm{~W}_{i j k \ell}^{+} \nabla_{i} \mathrm{~W}_{j m k \ell}+\nabla_{m} \mathrm{~W}_{i j m \ell}^{+}(d \mathrm{~A})_{i j \ell} .
$$

The splitting (3.10) implies $\delta \mathrm{W}=\delta \mathrm{W}^{+}+\delta \mathrm{W}^{-}$, so by (3.13)

$$
\nabla_{m} \mathrm{~W}_{i j m \ell}(d \mathrm{~A})_{i j \ell}=2\left(\delta \mathrm{W}^{+}\right)_{i j \ell}(\delta \mathrm{W})_{i j \ell}=2\left|\delta \mathrm{W}^{+}\right|^{2} .
$$

Similarly,

$$
-2 \nabla_{m} \mathrm{~W}_{i j k \ell}^{+} \nabla_{i} \mathrm{~W}_{j m k \ell}=-2 \nabla_{m} \mathrm{~W}_{i j k \ell}^{+} \nabla_{i} \mathrm{~W}_{j m k \ell}^{+} .
$$

Substituting these into (3.14) we obtain

$$
\int\left|\nabla \mathrm{W}^{+}\right|^{2}=\int-2 \nabla_{m} \mathrm{~W}_{i j k \ell}^{+} \nabla_{i} \mathrm{~W}_{j m k \ell}^{+}+2\left|\delta \mathrm{W}^{+}\right|^{2} .
$$

We analyze each term in (3.15) separately.

For the first term, we integrate by parts and commute derivatives:

$$
\begin{aligned}
& \int-2 \nabla_{m} \mathrm{~W}_{j k \ell}^{+} \nabla_{i} \mathrm{~W}_{j m k \ell}^{+}=\int 2 \mathrm{~W}_{j k \ell}^{+} \nabla_{m} \nabla_{i} \mathrm{~W}_{j m k \ell}^{+} \\
& =\int 2 \mathrm{~W}_{i j k \ell}^{+}\left\{\nabla_{i} \nabla_{m} \mathrm{~W}_{j m k \ell}^{+}+\mathrm{R}_{m i j s} \mathrm{~W}_{s m k \ell}^{+}\right. \\
& +\mathrm{R}_{\text {mims }} \mathrm{W}_{j s k \ell}^{+}+\mathrm{R}_{\text {miks }} \mathrm{W}_{j m s \ell}^{+} \\
& \left.+\mathrm{R}_{\text {mils }} \mathrm{W}_{j m k s}^{+}\right\} \\
& =\int 2 \mathrm{~W}_{i j k \ell}^{+} \nabla_{i} \nabla_{m} \mathrm{~W}_{j m k \ell}^{+}+2 \mathrm{R}_{\text {mijs }} \mathrm{W}_{i j k \ell}^{+} \mathrm{W}_{\text {smk }}^{+} \\
& +2 \mathrm{R}_{i s} \mathrm{~W}_{i j k \ell}^{+} \mathrm{W}_{j s k \ell}^{+}+2 \mathrm{R}_{m i k s} \mathrm{~W}_{i j k \ell}^{+} \mathrm{W}_{j m s \ell}^{+} \\
& +2 \mathrm{R}_{\text {mils }} \mathrm{W}_{i j k \ell}^{+} \mathrm{W}_{j m k s}^{+} \text {, }
\end{aligned}
$$


where $\mathrm{R}_{i s}=\mathrm{R}_{\text {mims }}$ are the components of the Ricci tensor. Note by re-indexing the last two terms in (3.16) are equal. If we integrate by parts again, the first term in (3.16) is

$$
\int 2 \mathrm{~W}_{j k \ell}^{+} \nabla_{i} \nabla_{m} \mathrm{~W}_{j m k \ell}^{+}=\int-2 \nabla_{i} \mathrm{~W}_{j k k \ell}^{+} \nabla_{m} \mathrm{~W}_{j m k \ell}^{+}=\int 2\left|\delta \mathrm{W}^{+}\right|^{2} .
$$

Using the Bianchi identity and re-indexing the next term can be rewritten

$$
2 \mathrm{R}_{m i j s} \mathrm{~W}_{i j k \ell}^{+} \mathrm{W}_{s m k \ell}^{+}=\mathrm{R}_{m s i j} \mathrm{~W}_{j k \ell}^{+} \mathrm{W}_{m s k \ell}^{+} \text {. }
$$

Appealing to the decomposition (1.0) once more, we get

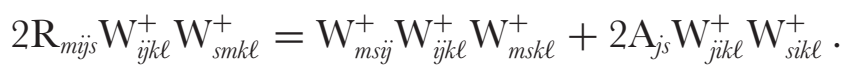

Similarly,

$$
4 \mathrm{R}_{m i k s} \mathrm{~W}_{j k \ell}^{+} \mathrm{W}_{j m s \ell}^{+}=4 \mathrm{~W}_{m i k s} \mathrm{~W}_{j k \ell}^{+} \mathrm{W}_{j m s \ell}^{+}+2 \mathrm{~A}_{i s} \mathrm{~W}_{j k \ell}^{+} \mathrm{W}_{j k s \ell}^{+}+2 \mathrm{~A}_{k m} \mathrm{~W}_{j k \ell}^{+} \mathrm{W}_{j m i \ell}^{+} \cdot
$$

Using the symmetries of the Weyl tensor and re-indexing we find

$$
2 \mathrm{~A}_{i s} \mathrm{~W}_{i j k \ell}^{+} \mathrm{W}_{j k s \ell}^{+}+2 \mathrm{~A}_{k m} \mathrm{~W}_{i j k \ell}^{+} \mathrm{W}_{j m i l}^{+}=-2 \mathrm{~A}_{k m} \mathrm{~W}_{i j \ell k}^{+} \mathrm{W}_{i j \ell m}^{+} .
$$

Combining (3.15)-(3.21),

$$
\begin{aligned}
\int\left|\nabla \mathrm{W}^{+}\right|^{2}=\int & 4\left|\delta \mathrm{W}^{+}\right|^{2}+2 \mathrm{R}_{i s} \mathrm{~W}_{i j k \ell}^{+} \mathrm{W}_{j s k \ell}^{+} \\
& +\mathrm{W}_{m s i j}^{+} \mathrm{W}_{j k \ell}^{+} \mathrm{W}_{m s k \ell}^{+}+4 \mathrm{~W}_{m i k s} \mathrm{~W}_{j k \ell}^{+} \mathrm{W}_{j m s \ell}^{+} .
\end{aligned}
$$

\section{Lemma 3.4.}

(i) $\mathrm{W}_{i j k \ell}^{+} \mathrm{W}_{j s k \ell}^{+}=-\frac{1}{4}\left|\mathrm{~W}^{+}\right|^{2} \delta_{i j}$,

(ii) $\mathrm{W}_{m s i j}^{+} \mathrm{W}_{i j k \ell}^{+} \mathrm{W}_{m s k \ell}^{+}=24 \operatorname{det} \mathrm{W}^{+}$,

(iii) $4 \mathrm{~W}_{\text {miks }} \mathrm{W}_{i j k \ell}^{+} \mathrm{W}_{j m s \ell}^{+}=48 \operatorname{det} \mathrm{W}^{+}$.

Proof. - As in [De], we fix a point and diagonalize $W^{ \pm}: \Lambda_{ \pm}^{2} \longrightarrow \Lambda_{ \pm}^{2}$. Let $\lambda_{i}^{ \pm}, 1 \leq i \leq 3$ denote the three eigenvalues of $\mathrm{W}^{ \pm}$, with corresponding eigenforms 
$\omega^{ \pm}, \eta^{ \pm}, \theta^{ \pm}$. Then

$$
\mathrm{W}^{ \pm}=\lambda_{1}^{ \pm} \omega^{ \pm} \otimes \omega^{ \pm}+\lambda_{2}^{ \pm} \eta^{ \pm} \otimes \eta^{ \pm}+\lambda_{3}^{ \pm} \theta^{ \pm} \otimes \theta^{ \pm}
$$

Then (i)-(iii) follow from elementry calculations.

From the preceding Lemma and (3.22) we obtain the identity

$$
\int\left|\nabla \mathrm{W}^{+}\right|^{2}=\int 4\left|\delta \mathrm{W}^{+}\right|^{2}+72 \operatorname{det} \mathrm{W}^{+}-\frac{1}{2} \mathrm{R}\left|\mathrm{W}^{+}\right|^{2},
$$

which holds for any Riemannian four-manifold.

Now suppose $\left(\mathrm{M}^{4}, g\right)$ is Bach-flat. By [De, (23)],

$$
0=\nabla_{k} \nabla_{\ell} \mathrm{W}_{i k \ell j}-\frac{1}{2} \mathrm{~W}_{i k j \ell} \mathrm{A}_{k \ell} .
$$

Pairing both sides with the Weyl-Schouten tensor and integrating we get

$$
\begin{aligned}
0 & =\int \mathrm{A}_{i j} \nabla_{k} \nabla_{\ell} \mathrm{W}_{i k \ell j}-\frac{1}{2} \mathrm{~W}_{i k j \ell} \mathrm{A}_{k \ell} \mathrm{A}_{i j} \\
& =\int-\nabla_{k} \mathrm{~A}_{i j} \nabla_{\ell} \mathrm{W}_{i k \ell j}-\frac{1}{2} \mathrm{~W}_{i k j \ell} \mathrm{A}_{k \ell} \mathrm{A}_{i j} \\
& =\int-\frac{1}{2}\left(\nabla_{k} \mathrm{~A}_{i j}-\nabla_{i} \mathrm{~A}_{k j}\right) \nabla_{\ell} \mathrm{W}_{i k \ell j}-\frac{1}{2} \mathrm{~W}_{i k j \ell} \mathrm{A}_{k \ell} \mathrm{A}_{i j} \\
& =\int-\frac{1}{2}(d \mathrm{~A})_{k i j} \nabla_{\ell} \mathrm{W}_{i k \ell j}-\frac{1}{2} \mathrm{~W}_{i k j \ell} \mathrm{A}_{k \ell} \mathrm{A}_{i j} \\
& =\int|\delta \mathrm{W}|^{2}-\frac{1}{2} \mathrm{~W}_{i k j \ell} \mathrm{A}_{k \ell} \mathrm{A}_{i j} .
\end{aligned}
$$

Since the Weyl tensor is trace-free, we conclude

$$
\int|\delta \mathrm{W}|^{2}=\int \frac{1}{2} \mathrm{~W}_{i j k \ell} \mathrm{E}_{i k} \mathrm{E}_{j \ell} .
$$

Combining (3.23) and (3.24), we get

$$
\begin{aligned}
\int|\nabla \mathrm{W}|^{2} & =\int\left|\nabla \mathrm{W}^{+}\right|^{2}+\int\left|\nabla \mathrm{W}^{-}\right|^{2} \\
& =\int 72 \operatorname{det} \mathrm{W}^{+}+72 \operatorname{det} \mathrm{W}^{-}-\frac{1}{2} \mathrm{R}|\mathrm{W}|^{2}+2 \mathrm{~W}_{i k \ell} \mathrm{E}_{i k} \mathrm{E}_{j \ell} .
\end{aligned}
$$

This completes the proof of Proposition 3.3. 
Combining (3.1), (3.7) and (3.8) we find that for any $\alpha \geq 0$,

$$
\begin{aligned}
& 0=\int_{\mathrm{M}^{4}}\left\{\frac{3}{2} \alpha|\nabla \mathrm{W}|^{2}+3\left(|\nabla \mathrm{E}|^{2}-\frac{1}{12}|\nabla \mathrm{R}|^{2}\right)\right. \\
& +6 t r \mathrm{E}^{3}+\mathrm{R}|\mathrm{E}|^{2}-3(\alpha+2) \mathrm{W}_{i j \kappa \ell} \mathrm{E}_{i \kappa} \mathrm{E}_{j \ell} \\
& \left.-108 \alpha \operatorname{det} \mathrm{W}^{+}-108 \alpha \operatorname{det} \mathrm{W}^{-}+\frac{3}{4} \alpha \mathrm{R}|\mathrm{W}|^{2}\right\} d v o l \\
& \geq \int_{\mathrm{M}^{4}} 6 t r \mathrm{E}^{3}+\mathrm{R}|\mathrm{E}|^{2}-3(\alpha+2) \mathrm{W}_{i j \kappa \ell} \mathrm{E}_{i \kappa} \mathrm{E}_{j \ell}
\end{aligned}
$$

$$
-108 \alpha \operatorname{det} \mathrm{W}^{+}-108 \alpha \operatorname{det} \mathrm{W}^{-}+\frac{3}{4} \alpha \mathrm{R}|\mathrm{W}|^{2} .
$$

This is the key identity in the proof of Theorem C.

\section{The proof of Theorem $\mathrm{C}$}

Suppose $\left(\mathrm{M}^{4}, g\right)$ is Bach-flat with positive Yamabe invariant, and that (0.6) holds:

$$
\int_{\mathrm{M}^{4}}|\mathrm{~W}|^{2} d v o l=16 \pi^{2} \chi\left(\mathrm{M}^{4}\right) \text {. }
$$

This is equivalent to

$$
\int_{\mathrm{M}^{4}} \sigma_{2}(\mathrm{~A}) d v o l=\frac{1}{4} \int_{\mathrm{M}^{4}}|\mathrm{~W}|^{2} d v o l .
$$

Now, if $\mathrm{W} \equiv 0$ then $\left(\mathrm{M}^{4}, g\right)$ is locally conformally flat and (by $\left.(0.6)\right) \chi\left(\mathrm{M}^{4}\right)=0$. It follows from $[\mathrm{Gu}$, Corollary $\mathrm{G}]$ that $\left(\mathrm{M}^{4}, g\right)$ is conformal to a manifold which is isometrically covered by $\mathrm{S}^{3} \times \mathrm{S}^{1}$. Therefore, let us assume from now on that

$$
\int_{\mathrm{M}^{4}}|\mathrm{~W}|^{2} d v o l>0 \text {. }
$$

By (4.1), this implies that for any $0 \leq \alpha<1$,

$$
\int_{\mathrm{M}^{4}} \sigma_{2}(\mathrm{~A}) d v o l-\frac{\alpha}{4} \int_{\mathrm{M}^{4}}|\mathrm{~W}|^{2} d v o l=\frac{(1-\alpha)}{4} \int_{\mathrm{M}^{4}}|\mathrm{~W}|^{2} d v o l>0 .
$$

According to Theorem 1.1, there is a conformal metric $g_{\alpha}=e^{2 \omega_{\alpha}} g$ satisfying

$$
\sigma_{2}\left(\mathrm{~A}_{g_{\alpha}}\right)-\frac{\alpha}{4}\left|\mathrm{~W}_{g_{\alpha}}\right|^{2} \equiv \lambda_{\alpha}>0 .
$$


Choose a sequence $\alpha_{\kappa} \nearrow 1$, and denote $g_{\kappa}=g_{\alpha_{\kappa}}=e^{2 \omega_{\kappa}} g$. The compactness properties of the sequence $\left\{g_{\kappa}\right\}$ require careful description.

First, we claim that the a priori estimate of [CGY2] holds: i.e., there is a constant $\mathrm{C}$ such that

$$
\left\|\nabla \omega_{\kappa}\right\|_{\infty}+\left\|\omega_{\kappa}\right\|_{\infty} \leq \mathrm{G} .
$$

(Recall (4.2) implies that $\left(\mathrm{M}^{4}, g\right)$ cannot be conformally equivalent to the sphere). This estimate is immediate from Proposition 1.7. In addition, the local estimate of [CGY2, Cor. 1.3] applies, and consequently we have a bound

$$
\left\|\nabla^{2} \omega_{\kappa}\right\|_{\infty} \leq \mathrm{G} .
$$

It is important to note that (4.5) is optimal: (4.3) is elliptic if $\sigma_{2}(\mathrm{~A})>0$; but $\lambda_{\kappa} \rightarrow 0$ as $\kappa \rightarrow \infty$, and there is no guarantee that the Weyl tensor does not vanish on $\mathbf{M}^{4}$. However, higher order estimates for $\left\{g_{\kappa}\right\}$ can be established on the set where the Weyl tensor is non-zero. To explain this, let

$$
\begin{aligned}
& \mathbf{M}_{+}^{4}=\left\{x \in \mathrm{M}^{4}:\left|\mathrm{W}_{g}\right|>0\right\}, \\
& \mathbf{M}_{0}^{4}=\left\{x \in \mathrm{M}^{4}:\left|\mathrm{W}_{g}\right|=0\right\} .
\end{aligned}
$$

By conformal invariance, the Weyl tensor of each $g_{\kappa}$ is also non-zero on $\mathbf{M}_{+}^{4}$ (and vanishes on $\left.\mathbf{M}_{0}^{4}\right)$. If $x_{0} \in \mathrm{M}_{+}^{4}$, then there are constants $\epsilon>0, \rho>0$, such that $\left|\mathrm{W}_{g}\right| \geq$ $\epsilon>0$ on the geodesic ball $\mathrm{B}_{\rho}\left(x_{0}\right)=\left\{x \in \mathrm{M}^{4}:\right.$ dist $\left.\left(x, x_{0}\right)<\rho\right\}$. On $\mathrm{B}_{\rho}\left(x_{0}\right)$ the metric $g_{\kappa}=e^{2 \omega \kappa} g$ satisfies

$$
\sigma_{2}\left(\mathrm{~A}_{g_{\kappa}}\right)=\frac{\alpha_{\kappa}}{4}\left|\mathrm{~W}_{g_{\kappa}}\right|^{2}+\lambda_{\kappa}=\frac{\alpha_{\kappa}}{4} e^{-4 \omega_{\kappa}}\left|\mathrm{W}_{g}\right|^{2}+\lambda_{\kappa} .
$$

In particular, by the a priori estimate (4.4) we see that

$$
\sigma_{2}\left(\mathrm{~A}_{g_{\kappa}}\right) \geq \mathrm{C}_{\epsilon}>0
$$

on $\mathrm{B}_{\rho}\left(x_{0}\right)$. Therefore,

$$
\sqrt{\sigma_{2}\left(\mathrm{~A}_{g_{\alpha}}\right)}=\sqrt{\frac{\alpha}{4}\left|\mathrm{~W}_{g_{\alpha}}\right|^{2}+\lambda_{\alpha}}
$$

is a strictly elliptic, concave equation on $\mathrm{B}_{\rho}\left(x_{0}\right)$. The regularity results of Evans [Ev] and Krylov [Kr] then give Hölder estimates for $\nabla^{2} \omega_{\kappa}$ on $\mathrm{B}_{\rho^{\prime}}\left(x_{0}\right)$ for any $\rho^{\prime}<\rho$ (see [GT, Theorem 17.14]). Applying Schauder theory and classic elliptic regularity, we then obtain estimates for derivatives of all orders on any ball $\mathrm{B}_{\rho^{\prime}}\left(x_{0}\right) \varsubsetneqq \mathrm{B}_{\rho}\left(x_{0}\right)$ with $\rho^{\prime}<\rho$. Consequently, a subsequence of $\left\{g_{\kappa}\right\}$ (also denoted $\left\{g_{\kappa}\right\}$ ) converges to a limiting metric $g_{\infty}$ in $\mathrm{C}_{l o c}^{\infty}\left(\mathrm{M}_{+}^{4}\right) \cap \mathrm{C}^{1,1}\left(\mathrm{M}^{4}\right)$. 
Recall that inequality (3.25) is satisfied by each $g_{\kappa}$ (with $\alpha=\alpha_{\kappa}$ ). If we split the integral in (3.25) into two integrals, one over $\mathbf{M}_{+}^{4}$, the other over $\mathbf{M}_{0}^{4}$, note that on $\mathbf{M}_{0}^{4}$ the integrand reduces to

$$
6 t r \mathrm{E}^{3}+\mathrm{R}|\mathrm{E}|^{2} .
$$

Using the sharp inequality $t r \mathrm{E}^{3} \geq-\frac{1}{\sqrt{3}}|\mathrm{E}|^{3}$, we find that

$$
6 t r \mathrm{E}^{3}+\mathrm{R}|\mathrm{E}|^{2} \geq-2 \sqrt{3}|\mathrm{E}|^{2}+\mathrm{R}|\mathrm{E}|^{2}=|\mathrm{E}|^{2}(\mathrm{R}-2 \sqrt{3}|\mathrm{E}|) .
$$

Since $\sigma_{2}(\mathrm{~A}) \geq 0$ on $\mathrm{M}_{0}^{4}$, we also have

$$
\begin{aligned}
0 & \leq-\frac{1}{2}|\mathrm{E}|^{2}+\frac{1}{24} \mathrm{R}^{2} \\
\Rightarrow \mathrm{R}^{2} & \geq 12|\mathrm{E}|^{2} \\
\Rightarrow \mathrm{R} & \geq 2 \sqrt{3}|\mathrm{E}| .
\end{aligned}
$$

Combining (4.6) and (4.7) we see that the integrand in (3.25) is non-negative on the set $\mathrm{M}_{0}^{4}$. Therefore, in view of the convergence of $\left\{g_{\kappa}\right\}$ on $\mathrm{M}_{+}^{4}$ we have

$$
\begin{aligned}
& 0 \geq \lim _{\kappa \rightarrow \infty} \int_{\mathrm{M}_{+}^{+}} 6 t r \mathrm{E}^{3}+\mathrm{R}|\mathrm{E}|^{2}-3\left(\alpha_{\kappa}+2\right) \mathrm{W}_{i j \kappa \ell} \mathrm{E}_{i \kappa} \mathrm{E}_{j \ell} \\
& \quad-108 \alpha_{\kappa} \operatorname{det} \mathrm{W}^{+}-108 \alpha_{\kappa} \operatorname{det} \mathrm{W}^{-}+\frac{3}{4} \alpha_{\kappa} \mathrm{R}|\mathrm{W}|^{2} \\
& =\int_{\mathrm{M}_{+}^{+}} 6 t r \mathrm{E}^{3}+\mathrm{R}|\mathrm{E}|^{2}-9 \mathrm{~W}_{i j \kappa \ell} \mathrm{E}_{i \kappa} \mathrm{E}_{j \ell}
\end{aligned}
$$

$$
-108 \operatorname{det} \mathrm{W}^{+}-108 \operatorname{det} \mathrm{W}^{-}+\frac{3}{4} \mathrm{R}|\mathrm{W}|^{2} .
$$

To summarize: we have constructed a metric $g_{\infty}=e^{2 w_{\infty}} g$ on $\mathrm{M}^{4}$ with $w_{\infty} \in$ $\mathrm{C}^{\infty}\left(\mathrm{M}_{+}^{4}\right) \cap \mathrm{C}^{1,1}\left(\mathrm{M}^{4}\right)$ which satisfies

$$
\begin{aligned}
& \sigma_{2}(\mathrm{~A})=\frac{1}{4}|\mathrm{~W}|^{2} \text { on } \mathrm{M}_{+}^{4}, \\
& 0 \geq \int_{\mathrm{M}_{+}^{4}}\left\{-108 \operatorname{det} \mathrm{W}^{+}-108 \operatorname{det} \mathrm{W}^{-}\right. \\
&+6 t r \mathrm{E}^{3}-9 \mathrm{~W}_{i j \kappa \ell} \mathrm{E}_{i \kappa} \mathrm{E}_{j \ell} \\
&\left.+\mathrm{R}\left(|\mathrm{E}|^{2}+\frac{3}{4}|\mathrm{~W}|^{2}\right)\right\} d v o l .
\end{aligned}
$$

Proposition 4.1. - If $g_{\infty}$ satisfies (4.9), then

(i) $g_{\infty} \in \mathrm{C}^{\infty}\left(\mathbf{M}^{4}\right)$;

(ii) $g_{\infty}$ is Einstein;

(iii) either $\mathrm{W}^{+} \equiv 0$ or $\mathrm{W}^{-} \equiv 0$ on $\mathrm{M}^{4}$. 
Proof. - Our first task is to rewrite the integrand in (4.9) in a suitable basis. To this end, let Riem : $\Lambda^{2} \rightarrow \Lambda^{2}$ denote the curvature operator of $\left(\mathrm{M}^{4}, g_{\infty}\right)$. Since $\mathrm{M}^{4}$ is oriented, we have the splitting $\Lambda^{2}=\Lambda_{+}^{2} \oplus \Lambda_{-}^{2}$, and the well known decomposition of Singer-Thorpe [ST]:

$$
\text { Riem }=\left(\begin{array}{ccc}
\mathrm{W}^{+}+\frac{1}{6} \mathrm{R} \mathrm{I} d & \vdots & \mathrm{B} \\
\ldots \ldots \ldots \ldots & \vdots & \ldots \ldots \ldots \ldots \\
\mathrm{B}^{\star} & \vdots & \mathrm{W}^{-}+\frac{1}{6} \mathrm{R} \mathrm{I} d
\end{array}\right)
$$

Note the compositions satisfy

$$
\begin{aligned}
& \mathrm{BB}^{\star}: \Lambda_{+}^{2} \rightarrow \Lambda_{+}^{2}, \\
& \mathrm{~B}^{\star} \mathrm{B}: \Lambda_{-}^{2} \rightarrow \Lambda_{-}^{2} .
\end{aligned}
$$

Fix a point $\mathrm{P} \in \mathrm{M}_{+}^{4}$, and let $\lambda_{1}^{ \pm} \leq \lambda_{2}^{ \pm} \leq \lambda_{3}^{ \pm}$denote the eigenvalues of $\mathrm{W}^{ \pm}$. Then

$$
\operatorname{det} \mathrm{W}^{ \pm}=\lambda_{1}^{ \pm} \lambda_{2}^{ \pm} \lambda_{3}^{ \pm}
$$

$$
\left|\mathrm{W}^{ \pm}\right|^{2}=4\left\|\mathrm{~W}^{ \pm}\right\|^{2}=4\left[\left(\lambda_{1}^{ \pm}\right)^{2}+\left(\lambda_{2}^{ \pm}\right)^{2}+\left(\lambda_{3}^{ \pm}\right)^{2}\right] .
$$

Recall that $\left\|\mathrm{W}^{ \pm}\right\|$denotes the norm of $\mathrm{W}^{ \pm}$when interpreted as an endomorphism of $\Lambda_{ \pm}^{2}$

Following Margerin, we denote the eigenvalues of $\mathrm{BB}^{\star}: \Lambda_{+}^{2} \rightarrow \Lambda_{+}^{2}$ by $b_{1}^{2} \leq b_{2}^{2} \leq b_{3}^{2}$, where $0 \leq b_{1} \leq b_{2} \leq b_{3}$.

\section{Lemma 4.2.}

$$
\begin{aligned}
& |\mathrm{E}|^{2}=4\left(b_{1}^{2}+b_{2}^{2}+b_{3}^{2}\right), \\
& t r \mathrm{E}^{3} \geq-24 b_{1} b_{2} b_{3} .
\end{aligned}
$$

Proof. - Given a basis $\left\{e_{i}\right\}$ of $\mathrm{T}_{p} \mathrm{M}^{4}$, let $\left\{e_{i}^{\star}\right\}$ denote the dual basis of $\mathrm{T}_{p}^{\star} \mathrm{M}^{4}$. Relative to this basis, the curvature operator is given by

$$
\operatorname{Riem}\left(e_{i}^{\star} \wedge e_{j}^{\star}\right)=\frac{1}{2} \sum_{\kappa, \ell} \mathrm{R}_{i j \kappa \ell} e_{\kappa}^{\star} \wedge e_{\ell}^{\star},
$$

where $\mathbf{R}_{i j k \ell}$ are components of Riem viewed as a $(0,4)$-tensor; i.e., $\mathbf{R}_{i j k \ell}=\operatorname{Riem}\left(e_{i}, e_{j}\right.$, $\left.e_{\kappa}, e_{\ell}\right)$. If $\mathrm{E}_{i j}=\mathrm{E}\left(e_{i}, e_{j}\right)$ are the components of the trace-free Ricci tensor, then the decomposition (0.1) implies

$$
\begin{array}{r}
\mathrm{R}_{i j \kappa \ell}=\mathrm{W}_{i j \kappa \ell}+\frac{1}{2}\left(\delta_{i \kappa} \mathrm{E}_{j \ell}-\delta_{i \ell} \mathrm{E}_{j \kappa}-\delta_{j \kappa} \mathrm{E}_{i \ell}+\delta_{j \ell} \mathrm{E}_{i \kappa}\right) \\
+\frac{1}{12} \mathrm{R}\left(\delta_{i \kappa} \delta_{j \ell}-\delta_{i \ell} \delta_{j \kappa}\right),
\end{array}
$$

where $\mathrm{W}_{i j \kappa \ell}=\mathrm{W}\left(e_{i}, e_{j}, e_{\kappa}, e_{\ell}\right)$. 
A basis $\left\{e_{i}^{\star}\right\}$ of $\mathrm{T}_{p}^{\star} \mathrm{M}^{4}$ induces a natural orthonormal basis of $\Lambda_{ \pm}^{2}$ :

$(\mathbf{4 . 1 8})$

$$
\begin{aligned}
\omega^{ \pm} & =\frac{1}{\sqrt{2}}\left(e_{1}^{\star} \wedge e_{2}^{\star} \pm e_{3}^{\star} \wedge e_{4}^{\star}\right), \\
\eta^{ \pm} & =\frac{1}{\sqrt{2}}\left(e_{1}^{\star} \wedge e_{3}^{\star} \mp e_{2}^{\star} \wedge e_{4}^{\star}\right), \\
\theta^{ \pm} & =\frac{1}{\sqrt{2}}\left(e_{1}^{\star} \wedge e_{4}^{\star} \pm e_{2}^{\star} \wedge e_{3}^{\star}\right) .
\end{aligned}
$$

Now suppose the basis $\left\{e_{i}\right\}$ diagonalizes $\mathrm{E}$ :

$$
\mathrm{E}=\left(\begin{array}{lllll}
\mathrm{E}_{11} & & & & 0 \\
& \mathrm{E}_{22} & & & \\
& & \mathrm{E}_{33} & & \\
0 & & & \mathrm{E}_{44}
\end{array}\right) .
$$

Using (4.11), (4.16), and (4.17), the matrix of $\mathrm{B}: \Lambda_{-}^{2} \rightarrow \Lambda_{+}^{2}$ relative to the basis in $(4.18)$ is

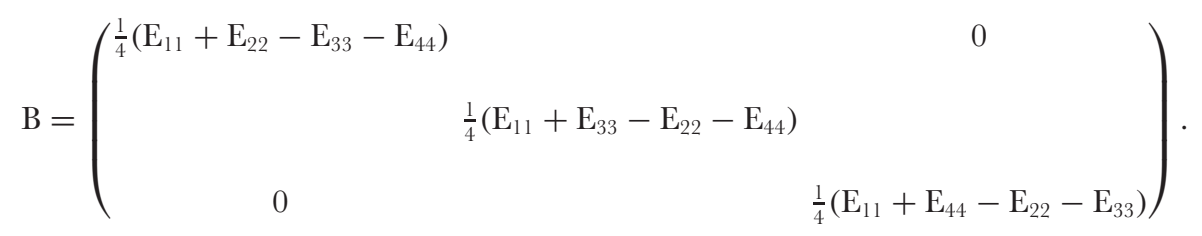

Let

$$
\begin{aligned}
& \mu_{1}=\frac{1}{4}\left(\mathrm{E}_{11}+\mathrm{E}_{22}-\mathrm{E}_{33}-\mathrm{E}_{44}\right), \\
& \mu_{2}=\frac{1}{4}\left(\mathrm{E}_{11}+\mathrm{E}_{33}-\mathrm{E}_{22}-\mathrm{E}_{44}\right), \\
& \mu_{3}=\frac{1}{4}\left(\mathrm{E}_{11}+\mathrm{E}_{44}-\mathrm{E}_{22}-\mathrm{E}_{33}\right) .
\end{aligned}
$$

Since $\mathrm{E}$ is trace-free, these can also be expressed

$$
\begin{aligned}
& \mu_{1}=\frac{1}{2}\left(\mathrm{E}_{11}+\mathrm{E}_{22}\right), \\
& \mu_{2}=\frac{1}{2}\left(\mathrm{E}_{11}+\mathrm{E}_{33}\right), \\
& \mu_{3}=\frac{1}{2}\left(\mathrm{E}_{11}+\mathrm{E}_{44}\right) .
\end{aligned}
$$

Consequently, in terms of $\left\{\mu_{1}, \mu_{2}, \mu_{3}\right\}$ the eigenvalues of $\mathrm{BB}^{\star}: \Lambda_{+}^{2} \rightarrow \Lambda_{+}^{2}$ are

$$
\begin{aligned}
& b_{1}^{2}=\mu_{1}^{2}, \\
& b_{2}^{2}=\mu_{2}^{2}, \\
& b_{3}^{2}=\mu_{3}^{2} .
\end{aligned}
$$

Now, a simple calculation gives

$$
\begin{aligned}
8 \mu_{1} \mu_{2} \mu_{3}= & \left(\mathrm{E}_{11}+\mathrm{E}_{22}\right)\left(\mathrm{E}_{11}+\mathrm{E}_{33}\right)\left(\mathrm{E}_{11}+\mathrm{E}_{44}\right) \\
= & \mathrm{E}_{11}^{3}+\mathrm{E}_{11}^{2} \mathrm{E}_{22}+\mathrm{E}_{11}^{2} \mathrm{E}_{33}+\mathrm{E}_{11}^{2} \mathrm{E}_{44} \\
& \quad+\mathrm{E}_{11} \mathrm{E}_{22} \mathrm{E}_{33}+\mathrm{E}_{11} \mathrm{E}_{22} \mathrm{E}_{44}+\mathrm{E}_{11} \mathrm{E}_{33} \mathrm{E}_{44}+\mathrm{E}_{22} \mathrm{E}_{33} \mathrm{E}_{44} \\
= & \mathrm{E}_{11}^{2}\left(\mathrm{E}_{11}+\mathrm{E}_{22}+\mathrm{E}_{33}+\mathrm{E}_{44}\right)+\sigma_{3}\left(\mathrm{E}_{11}, \mathrm{E}_{22}, \mathrm{E}_{33}, \mathrm{E}_{44}\right) .
\end{aligned}
$$


On the other hand, for a symmetric trace-free $4 \times 4$ matrix $\mathrm{E}, \sigma_{3}(\mathrm{E})=\frac{1}{3} t \mathrm{E}^{3}$. Thus

$$
\begin{aligned}
t r \mathrm{E}^{3} & =24 \mu_{1} \mu_{2} \mu_{3} \\
& \geq-24\left|\mu_{1} \mu_{2} \mu_{3}\right| \\
& =-24 b_{1} b_{2} b_{3} .
\end{aligned}
$$

This proves (4.15). The proof of (4.14) follows from (4.20), and will be omitted.

The next inequality follows from Lemma 6 in [Ma2]. However, as our notation and conventions are slightly different we provide some details.

\section{Lemma 4.3.}

$$
-\mathrm{W}_{i j \kappa \ell} \mathrm{E}_{i \kappa} \mathrm{E}_{j \ell} \geq-4\left[\sum_{i=1}^{3} \lambda_{i}^{+} b_{i}^{2}+\sum_{i=1}^{3} \lambda_{i}^{-} b_{i}^{2}\right] .
$$

Proof. - This inequality is termed "decoupling of the Weyl and Ricci curvatures" by Margerin, and appropriately enough: In general $\mathrm{W}^{ \pm}$and $\mathrm{BB}^{\star}$ or $\mathrm{B}^{\star} \mathrm{B}$ do not commute, and therefore cannot be simultaneously diagonalized.

In any case, if we choose a basis $\left\{e_{i}\right\}$ of $\mathrm{T}_{p} \mathrm{M}^{4}$ which diagonalizes $\mathrm{E}$ as in Lemma 1 , then the matrix of $\mathrm{W}^{ \pm}: \Lambda_{ \pm}^{2} \rightarrow \Lambda_{ \pm}^{2}$ relative to the basis in (4.18) is

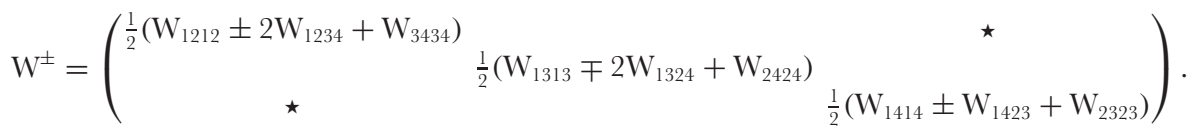

Therefore, if $\langle,\rangle_{\Lambda_{ \pm}^{2}}$ denotes the natural inner product induced on $\Lambda_{ \pm}^{2}$ (see Remark 2 in the Introduction), then

$$
\begin{aligned}
\left\langle\mathrm{W}^{+}, \mathrm{BB}^{\star}\right\rangle_{\Lambda_{+}^{2}}+\left\langle\mathrm{W}^{-}, \mathrm{B}^{\star} \mathrm{B}\right\rangle_{\Lambda_{-}^{2}}=\operatorname{tr} & \left(\mathrm{W}^{+} \circ \mathrm{BB}^{\star}+\mathrm{W}^{-} \circ \mathrm{B}^{\star} \mathrm{B}\right) \\
=\{ & \left(\mathrm{W}_{1212}+\mathrm{W}_{3434}\right) \mu_{1}^{2} \\
& +\left(\mathrm{W}_{1313}+\mathrm{W}_{2424}\right) \mu_{2}^{2} \\
& \left.+\left(\mathrm{W}_{1414}+\mathrm{W}_{2323}\right) \mu_{3}^{2}\right\} \\
=\frac{1}{2} & \left(\mathrm{~W}_{1212} \mathrm{E}_{11} \mathrm{E}_{22}+\mathrm{W}_{1313} \mathrm{E}_{11} \mathrm{E}_{33}\right. \\
& +\mathrm{W}_{1414} \mathrm{E}_{11} \mathrm{E}_{44}+\mathrm{W}_{2323} \mathrm{E}_{22} \mathrm{E}_{33} \\
& \left.+\mathrm{W}_{2424} \mathrm{E}_{22} \mathrm{E}_{44}+\mathrm{W}_{3434} \mathrm{E}_{33} \mathrm{E}_{44}\right) .
\end{aligned}
$$

On the other hand,

$$
\begin{aligned}
\mathrm{W}_{i j \kappa} \mathrm{E}_{i \kappa} \mathrm{E}_{j \ell}=2\left(\mathrm{~W}_{1212} \mathrm{E}_{11} \mathrm{E}_{22}+\mathrm{W}_{1313} \mathrm{E}_{11} \mathrm{E}_{33}+\mathrm{W}_{1414} \mathrm{E}_{11} \mathrm{E}_{44}\right. \\
\left.+\mathrm{W}_{2323} \mathrm{E}_{22} \mathrm{E}_{33}+\mathrm{W}_{2424} \mathrm{E}_{22} \mathrm{E}_{44}+\mathrm{W}_{3434} \mathrm{E}_{33} \mathrm{E}_{44}\right) .
\end{aligned}
$$


Therefore,

$$
-\mathrm{W}_{i j \kappa \ell} \mathrm{E}_{i \kappa} \mathrm{E}_{j \ell}=-4\left\langle\mathrm{~W}^{+}, \mathrm{BB}^{\star}\right\rangle_{\Lambda_{+}^{2}}-4\left\langle\mathrm{~W}_{1}^{-} \mathrm{B}^{\star} \mathrm{B}\right\rangle_{\Lambda_{-}^{2}} .
$$

According to Lemma 6 of [Ma2],

$$
\left\langle\mathrm{W}^{+}, \mathrm{BB}^{\star}\right\rangle_{\Lambda_{+}^{2}}+\left\langle\mathrm{W}^{-}, \mathrm{B}^{\star} \mathrm{B}\right\rangle_{\Lambda_{-}^{2}} \leq \sum_{i=1}^{3} \lambda_{i}^{+} b_{i}^{2}+\sum_{i=1}^{3} \lambda_{i}^{-} b_{i}^{2} .
$$

Combining (4.22) and (4.23) we obtain (4.21).

On the set $\mathrm{M}_{+}^{4}, g_{\infty}$ satisfies

$$
\sigma_{2}(\mathrm{~A})=\frac{1}{4}|\mathrm{~W}|^{2}=\|\mathrm{W}\|^{2}=\left\|\mathrm{W}^{+}\right\|^{2}+\mid \mathrm{W}^{-} \|^{2} .
$$

Therefore,

$$
\mathrm{R}=\sqrt{12|\mathrm{E}|^{2}+24\left\|\mathrm{~W}^{+}\right\|^{2}+24\left\|\mathrm{~W}^{-}\right\|^{2}} .
$$

Combining (4.21) and (4.24), the integrand in (4.9) at the point $\mathrm{P}$ satisfies the inequality

$$
\begin{aligned}
& -108 \operatorname{det} \mathrm{W}^{+}-108 \operatorname{det} \mathrm{W}^{-}+6 t r \mathrm{E}^{3}-9 \mathrm{~W}_{i j \kappa} \ell \mathrm{E}_{i \kappa} \mathrm{E}_{j \ell}+\mathrm{R}\left(|\mathrm{E}|^{2}+\frac{3}{4}|\mathrm{~W}|^{2}\right) \\
& \geq-108 \lambda_{1}^{+} \lambda_{2}^{+} \lambda_{3}^{+}-108 \lambda_{1}^{-} \lambda_{2}^{-} \lambda_{3}^{-}-144 b_{1} b_{2} b_{3} \\
& -36\left[\lambda_{1}^{+} b_{1}^{2}+\lambda_{2}^{+} b_{2}^{2}+\lambda_{3}^{+} b_{3}^{2}+\lambda_{1}^{-} b_{1}^{2}+\lambda_{2}^{-} b_{2}^{2}+\lambda_{3}^{-} b_{3}^{2}\right] \\
& +\left\{48\left(b_{1}^{2}+b_{2}^{2}+b_{3}^{2}\right)\right. \\
& \left.+24\left[\left(\lambda_{1}^{+}\right)^{2}+\left(\lambda_{2}^{+}\right)^{2}+\left(\lambda_{3}^{+}\right)^{2}+\left(\lambda_{1}^{-}\right)^{2}+\left(\lambda_{2}^{-}\right)^{2}+\left(\lambda_{3}^{-}\right)^{2}\right]\right\}^{\frac{1}{2}} \\
& \times\left\{4\left(b_{1}^{2}+b_{2}^{2}+b_{3}^{2}\right)\right. \\
& \left.+3\left[\left(\lambda_{1}^{+}\right)^{2}+\left(\lambda_{2}^{+}\right)^{2}+\left(\lambda_{3}^{+}\right)^{2}+\left(\lambda_{1}^{-}\right)^{2}+\left(\lambda_{2}^{-}\right)^{2}+\left(\lambda_{3}^{-}\right)^{2}\right]\right\} \\
& \equiv \mathrm{F}\left(\lambda_{1}^{+}, \lambda_{2}^{+}, \lambda_{3}^{+}, \lambda_{1}^{-}, \lambda_{2}^{-}, \lambda_{3}^{-}, b_{1}, b_{2}, b_{3}\right) \text {. }
\end{aligned}
$$

Proposition 4.4. - Suppose $0 \leq b_{1} \leq b_{2} \leq b_{3}, \lambda_{1}^{ \pm} \leq \lambda_{2}^{ \pm} \leq \lambda_{3}^{ \pm}$with $\sum_{i=1}^{3} \lambda_{i}^{ \pm}=0$ and $\sum_{i=1}^{3}\left(\left|\lambda_{i}^{+}\right|^{2}+\left|\lambda_{i}^{-}\right|^{2}\right) \neq 0$. Then $\mathrm{F}\left(\lambda_{1}^{+}, \lambda_{2}^{+}, \lambda_{3}^{+}, \lambda_{1}^{-}, \lambda_{2}^{-}, \lambda_{3}^{-}, b_{1}, b_{2}, b_{3}\right) \geq 0$, and equality holds if and only if one of the following is true:

(1) $b_{1}=b_{2}=b_{3}=0$ and there exists some $a \geq 0$ with $\lambda_{1}^{+}=\lambda_{2}^{+}=-a, \lambda_{3}^{+}=2 a$, $\lambda_{1}^{-}=\lambda_{2}^{-}=\lambda_{3}^{-}=0$; or $\lambda_{1}^{+}=-2 a, \lambda_{2}^{+}=\lambda_{3}^{+}=a, \lambda_{1}^{-}=\lambda_{2}^{-}=\lambda_{3}^{-}=0$; or similar cases with the role of $\lambda_{i}^{+}$and $\lambda_{i}^{-}$interchanged.

(2) $b_{1}=b_{2}=b_{3}, \lambda_{i}^{ \pm}=0$ for all $1 \leq i \leq 3$. 
The proof of Proposition 4.4 is given in the Appendix, and amounts to a complicated Lagrange-multiplier problem. We will assume the result for now, and explain how Proposition 4.1 follows.

By Proposition 4.4, the integrand in (4.9) is non-negative. Since the integral is less than or equal to zero, it follows that the integrand $\mathrm{F}\left(\lambda_{1}^{+}, \lambda_{2}^{+}, \lambda_{3}^{+}, \lambda_{1}^{-}, \lambda_{2}^{-}, \lambda_{3}^{-}\right.$, $\left.b_{1}, b_{2}, b_{3}\right) \equiv 0$. Thus, at each point in $\mathrm{M}_{+}^{4}$ either case (1) or case (2) of Proposition 4.4 above must hold. Since by definition $|\mathrm{W}|>0$ on $\mathrm{M}_{+}^{4}$, case (1) is the only possibility. In particular, $\mathrm{E} \equiv 0$ on $\mathrm{M}_{+}^{4}$ and at each point either $\mathrm{W}^{+}=0$ or $\mathrm{W}^{-}=0$.

Since $\mathrm{E} \equiv 0$ on $\mathrm{M}_{+}^{4}$ the scalar curvature is constant on each component of $\mathrm{M}_{+}^{4}$, which implies by (4.9) that $|\mathrm{W}|^{2}$ is also constant on each component. We claim that $\mathrm{M}_{+}^{4}=\mathrm{M}^{4}$; i.e., $\mathrm{M}_{0}^{4}$ is empty. To see why, choose a component $\mathrm{O}$ of $\mathrm{M}_{+}^{4}$ and a sequence of points $\left\{x_{i}\right\}$ in $\mathrm{O}$ with $x_{i} \rightarrow x_{0} \in \mathrm{M}_{0}^{4}$. Since $|\mathrm{W}|^{2}$ is constant in $\mathrm{O}$,

$$
c=\left|\mathrm{W}_{g_{\infty}}\right|^{2}\left(x_{i}\right)
$$

for some $c>0$. By conformal invariance of the Weyl tensor,

$$
c=\left|\mathbf{W}_{g_{\infty}}\right|^{2}\left(x_{i}\right)=e^{-4 w_{\infty}\left(x_{i}\right)}\left|\mathbf{W}_{g}\right|^{2}\left(x_{i}\right)
$$

By definition, $\left|\mathrm{W}_{g}\right|^{2}\left(x_{0}\right)=0$, and consequently $w_{\infty}\left(x_{i}\right) \rightarrow-\infty$ as $i \rightarrow \infty$. But this contradicts the fact that $w_{\infty} \in \mathrm{C}^{1,1}$. It follows that the Weyl tensor cannot vanish on $\mathbf{M}^{4}$, so $\left(\mathrm{M}^{4}, g_{\infty}\right)$ is a smooth Einstein manifold. Moreover, since $|\mathrm{W}|^{2}$ is constant and either $\mathrm{W}^{+}=0$ or $\mathrm{W}^{-}=0$ at each point, it follows that one of the components of the Weyl tensor vanishes identically on $\mathrm{M}^{4}$. By Hitchin's classification result $[\mathrm{Hi}],\left(\mathrm{M}^{4}, g_{\infty}\right)$ is homothetically isometric to $\pm \mathbf{C} \mathbf{P}^{2}$ with the Fubini-Study metric. This completes the proof of Proposition 4.1.

\section{Appendix}

In this appendix we establish Proposition A below, which is slightly more general than Proposition 4.4 .

Denote $\overrightarrow{\mathrm{B}}=\left(b_{1}, b_{2}, b_{3}\right), \overrightarrow{\mathrm{X}}=\left(x_{1}, x_{2}, x_{3}\right), \overrightarrow{\mathrm{Y}}=\left(y_{1}, y_{2}, y_{3}\right)$ vectors in $\mathbf{R}^{3}$, and $|\overrightarrow{\mathrm{B}}|^{2}=\sum_{i=1}^{3} b_{i}^{2},|\overrightarrow{\mathrm{X}}|^{2}=\sum_{i=1}^{3} x_{i}^{2},|\overrightarrow{\mathrm{Y}}|^{2}=\sum_{i=1}^{3} y_{i}^{2}$. Define the functional $\mathrm{I}=\mathrm{I}(\overrightarrow{\mathrm{B}}, \overrightarrow{\mathrm{X}}, \overrightarrow{\mathrm{Y}})$ by

$$
\begin{aligned}
\mathrm{I}= & \sqrt{6}\left[4|\overrightarrow{\mathrm{B}}|^{2}+3\left(|\overrightarrow{\mathrm{X}}|^{2}+|\overrightarrow{\mathrm{Y}}|^{2}\right)\right]\left(2|\overrightarrow{\mathrm{B}}|^{2}+|\overrightarrow{\mathrm{X}}|^{2}+|\overrightarrow{\mathrm{Y}}|^{2}\right)^{1 / 2} \\
& -54 x_{1} x_{2} x_{3}-54 y_{1} y_{2} y_{3}-72 b_{1} b_{2} b_{3} \\
& -18\left(x_{1} b_{1}^{2}+x_{2} b_{2}^{2}+x_{3} b_{3}^{2}+y_{1} b_{1}^{2}+y_{2} b_{2}^{2}+y_{3} b_{3}^{2}\right) .
\end{aligned}
$$


Proposition A. - Assume $b_{1}^{2} \leq b_{2}^{2} \leq b_{3}^{2}, \sum_{i=1}^{3} x_{i}=0, \sum_{i=1}^{3} y_{i}=0$. Then $\mathrm{I} \geq 0$. Further, I $=0$ only at the following points:

(i) $\overrightarrow{\mathrm{B}}=\overrightarrow{\mathrm{X}}=\overrightarrow{\mathrm{Y}}=(0,0,0)$, or

(ii) $\overrightarrow{\mathrm{B}}=(0,0,0)$ and either $\overrightarrow{\mathrm{X}}=(-a,-a, 2 a), \overrightarrow{\mathrm{Y}}=(0,0,0)$, or a permutation of $x_{1}=-a, x_{2}=-a, x_{3}=2 a$ and $\overrightarrow{\mathrm{Y}}=(0,0,0)$, or with the preceding values with the roles of $\overrightarrow{\mathrm{X}}$ and $\overrightarrow{\mathrm{Y}}$ reversed for some $a \neq 0$, or

(iii) $\overrightarrow{\mathrm{B}}=(b, b, b)$ for some $b \neq 0$ and $\overrightarrow{\mathrm{X}}=\overrightarrow{\mathrm{Y}}=(0,0,0)$.

Remark. - If we set $\mathrm{F}=2 \mathrm{I}, x_{i}=\lambda_{i}^{+}, y_{i}=\lambda_{i}^{-}(1 \leq i \leq 3)$, with $|\overrightarrow{\mathrm{X}}|^{2}+|\overrightarrow{\mathrm{Y}}|^{2} \neq 0$, then Proposition 4.4 is a consequence of Proposition A.

We will first establish Proposition $\mathrm{A}$ in the special case where $\overrightarrow{\mathrm{B}}=(0,0,0)$.

Lemma 1. - Denote

$$
\begin{aligned}
J(\vec{X}, \vec{Y})= & I(0, \vec{X}, \vec{Y}) \\
= & 3 \sqrt{6}\left(|\vec{X}|^{2}+|\vec{Y}|^{2}\right)\left(|\vec{X}|^{2}+|\vec{Y}|^{2}\right)^{1 / 2} \\
& -54 x_{1} x_{2} x_{3}-54 y_{1} y_{2} y_{3}
\end{aligned}
$$

with $\sum_{i=1}^{3} x_{i}=\sum_{i=1}^{3} y_{i}=0$. Then $\mathrm{J} \geq 0$, and $\mathrm{J}=0$ only when $\overrightarrow{\mathrm{X}}=\overrightarrow{\mathrm{Y}}=(0,0,0)$ or at the points

$$
\begin{array}{ll}
\overrightarrow{\mathrm{X}}=(-a,-a, 2 a), & \overrightarrow{\mathrm{Y}}=(0,0,0), \text { or } \\
\overrightarrow{\mathrm{X}}=(2 a,-a,-a), & \overrightarrow{\mathrm{Y}}=(0,0,0), \text { or } \\
\overrightarrow{\mathrm{X}}=(-a, 2 a,-a), & \overrightarrow{\mathrm{Y}}=(0,0,0) ;
\end{array}
$$

or with the roles of $\overrightarrow{\mathrm{X}}$ and $\overrightarrow{\mathrm{Y}}$ reversed, for some $a \neq 0$.

Proof. - In the case $\vec{X}=\vec{Y}=(0,0,0), J=0$. In the case $|\vec{X}|^{2}+|\vec{Y}|^{2} \neq 0$, replacing $x_{i}$ and $y_{i}$ by $\frac{ \pm x_{i}}{\left(|x|^{2}+|y|^{2}\right)^{1 / 2}}$ and $\frac{ \pm y_{i}}{\left(|x|^{2}+|y|^{2}\right)^{1 / 2}}$, we may assume w.l.o.g. that $|\overrightarrow{\mathrm{X}}|^{2}+$ $|\overrightarrow{\mathrm{Y}}|^{2}=1, x_{1} \leq x_{2} \leq x_{3}$, and $y_{1} \leq y_{2} \leq y_{3}$.

To find the minimal points of $\mathrm{J}$ we use the method of Lagrange multipliers. Let $\varphi_{1}=|\overrightarrow{\mathrm{X}}|^{2}+|\overrightarrow{\mathrm{Y}}|^{2}=1, \varphi_{2}=x_{1}+x_{2}+x_{3}=0$, and $\varphi_{3}=y_{1}+y_{2}+y_{3}=0$ denote the constraints, and let $\mu, 2 \beta, 2 \gamma$ denote the respective Lagrange multipliers. Set $\frac{\partial J}{\partial x_{i}}=$ $\mu \frac{\partial \varphi_{1}}{\partial x_{i}}+2 \beta \frac{\partial \varphi_{2}}{\partial x_{i}}, \frac{\partial J}{\partial y_{i}}=\mu \frac{\partial \varphi_{1}}{\partial y_{i}}+2 \gamma \frac{\partial \varphi_{3}}{\partial y_{i}}$ for $i=1,2,3$; we get the equations
(A1)
$-27 x_{2} x_{3}=\mu x_{1}+\beta$
(A2)
$-27 x_{1} x_{3}=\mu x_{2}+\beta$
(A3)
$-27 x_{1} x_{2}=\mu x_{3}+\beta$. 
Subtracting (A2) from (A1) we get

$$
-27\left(x_{2}-x_{1}\right) x_{3}=\mu\left(x_{1}-x_{2}\right),
$$

so we have either $x_{1}=x_{2}$, or $x_{1} \neq x_{2}$ while $27 x_{3}=\mu$. Subtracting (A3) from (A2) we get similarly $x_{2}=x_{3}$ or $x_{2} \neq x_{3}$ while $27 x_{1}=\mu$. Thus, we have three possibilities: either $x_{1}=x_{2}=x_{3}=0$, or $x_{1}=x_{2} \neq x_{3}$ with $27 x_{3}=\mu$, or $x_{1} \neq x_{2}=x_{3}$ with $27 x_{1}=\mu$. In summary, we have $\overrightarrow{\mathrm{X}}=(0,0,0)$ or $\overrightarrow{\mathrm{X}}=(-a,-a, 2 a)$ with $54 a=\mu$; or $\overrightarrow{\mathrm{X}}=(2 a,-a,-a)$ with $54 a=\mu$, where $a \geq 0$. By symmetry, we also have either $\overrightarrow{\mathrm{Y}}=(0,0,0)$, or $\overrightarrow{\mathrm{Y}}=(-c,-c, 2 c)$ with $54 c=\mu$, or $\overrightarrow{\mathrm{Y}}=(-2 c, c, c)$ with $-54 c=\mu$, where $c \geq 0$.

Combining the possibilities for $\overrightarrow{\mathrm{X}}$ and $\overrightarrow{\mathrm{Y}}$, we have eight cases.

(i) $\overrightarrow{\mathrm{X}}=(0,0,0), \quad \overrightarrow{\mathrm{Y}}=(-c,-c, 2 c)$; then $\mathrm{J}=0$.

(ii) $\overrightarrow{\mathrm{X}}=(0,0,0), \quad \overrightarrow{\mathrm{Y}}=(-2 c, c, c)$; then $\mathrm{J}=0$.

(iii) $\overrightarrow{\mathrm{X}}=(-a,-a, 2 a), \quad \overrightarrow{\mathrm{Y}}=(-c,-c, 2 c)$ with $54 a=\mu=54 c \geq 0$; $\quad$ then $\mathrm{J}(\overrightarrow{\mathrm{X}}, \overrightarrow{\mathrm{Y}})=3 \sqrt{6} \cdot 12 a^{2} \sqrt{12} a-54 \cdot 4 a^{3}=216(\sqrt{2}-1) a^{3} \geq 0$.

(iv) $\overrightarrow{\mathrm{X}}=(-a,-a, 2 a), \overrightarrow{\mathrm{Y}}=(-2 c, c, c)$ with $54 a=\mu=-54 c$; $\quad$ then $a=-c$ while both $a \geq 0, c \geq 0$; thus $\overrightarrow{\mathrm{X}}=\overrightarrow{\mathrm{Y}}=(0,0,0)$ and $\mathrm{J}=0$.

Cases $(\mathrm{v}) \rightarrow$ (viii) are similar to cases (ii) to (vi) with the roles of $\overrightarrow{\mathrm{X}}$ and $\overrightarrow{\mathrm{Y}}$ reversed. This finishes the proof of Lemma 1.

We now consider the general case in Proposition A. The proof is more tedious, but follows the same pattern of the proof of Lemma 1. We first outline the steps.

Outline of the proof of Proposition A. - When $\overrightarrow{\mathrm{B}}=(0,0,0)$, we apply Lemma 1 . So assume $\overrightarrow{\mathrm{B}} \neq(0,0,0)$. We also assume w.l.o.g. that $2|\overrightarrow{\mathrm{B}}|^{2}+|\overrightarrow{\mathrm{X}}|^{2}+|\overrightarrow{\mathrm{Y}}|^{2}=1$. To locate the minimal points of I under this constraint, we once again apply the method of Lagrange multipliers. We will break the proof into the following four steps:

Step 1. We may assume w.l.o.g. that $0 \leq b_{1} \leq b_{2} \leq b_{3}$ and $x_{1} \leq x_{2} \leq x_{3}$, $y_{1} \leq \overline{y_{2} \leq y_{3}}$, and $x_{3} \geq 0, y_{3} \geq 0$.

Step 2. Actually, $b_{1}=b_{2}=b$.

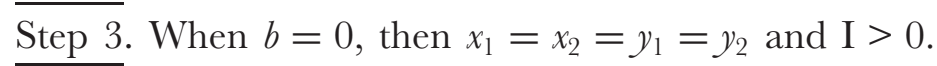

Step 4. When $b>0$, then $x_{1}=x_{2}=y_{1}=y_{2}=-a$, and either $a=0, b_{3}=b$, and $\mathrm{I} \overline{=0 ;}$ or $a>0$ and $\mathrm{I}>0$.

We now prove each of the steps in more detail.

Proof of Step 1. - We first observe that at a minimal point of $\mathrm{I}=\mathrm{I}(\overrightarrow{\mathrm{B}}, \overrightarrow{\mathrm{X}}, \overrightarrow{\mathrm{Y}})$ we may assume $b_{1} b_{2} b_{3} \geq 0$. Thus by switching $b_{i}$ with $-b_{i}(i=1,2,3)$, we may assume under the hypothesis $b_{1}^{2} \leq b_{2}^{2} \leq b_{3}^{2}$ that $0 \leq b_{1} \leq b_{1} \leq b_{3}$. 
Next observe for any $\overrightarrow{\mathrm{B}}$, for $\overrightarrow{\mathrm{X}}$ to be a minimal point of I we must have $x_{1} b_{1}^{2}+$ $x_{2} b_{2}^{2}+x_{3} b_{3}^{2} \leq x_{2} b_{1}^{2}+x_{1} b_{2}^{2}+x_{3} b_{3}^{2}$. Therefore, $\left(x_{1}-x_{2}\right) b_{1}^{2} \leq\left(x_{1}-x_{2}\right) b_{2}^{2}$. Thus unless $b_{1}^{2}=b_{2}^{2}=0$, we have $x_{1} \leq x_{2}$; but when $b_{1}^{2}=b_{2}^{2}=0$, we may also switch the order of $x_{1}$ and $x_{2}$ if necessary, and assume $x_{1} \leq x_{2}$ to attain the same value of I. Thus we can argue similarly and obtain $x_{1} \leq x_{2} \leq x_{3}$ and $y_{1} \leq y_{2} \leq y_{3}$. Since $\sum_{i=1}^{3} x_{i}=\sum_{i=1}^{3} y_{i}=0$, it follows that $x_{3} \geq 0, y_{3} \geq 0$.

Proof of Step 2. - We now set up the Lagrange multiplier problem under the constraints

$$
\begin{array}{r}
\varphi_{1}=2|\overrightarrow{\mathrm{B}}|^{2}+|\overrightarrow{\mathrm{X}}|^{2}+|\overrightarrow{\mathrm{Y}}|^{2}=1 \\
\varphi_{2}=x_{1}+x_{2}+x_{3}=0 \\
\varphi_{3}=y_{1}+y_{2}+y_{3}=0
\end{array}
$$

with multipliers $\mu, 2 \beta, 2 \gamma$, respectively. To locate the minimal point(s) of I under the given constraints we set $\frac{\partial \mathrm{I}}{\partial b_{i}}=\mu \frac{\partial \varphi_{1}}{\partial b_{i}}, \frac{\partial \mathrm{I}}{\partial x_{i}}=\mu \frac{\partial \varphi_{1}}{\partial x_{i}}+2 \beta \frac{\partial \varphi_{2}}{\partial x_{i}}, \frac{\partial \mathrm{I}}{\partial y_{i}}=\mu \frac{\partial \varphi_{1}}{\partial y_{i}}+2 \gamma \frac{\partial \varphi_{3}}{\partial y_{i}}$ for $i=$ 1, 2, 3 and obtain the following nine equations:

(A4)

(A5)

(A6)

(A7)

(A8)

(A9)

$$
\begin{aligned}
-9\left(x_{1}+y_{1}\right) b_{1}-18 b_{2} b_{3} & =(\mu-2 \sqrt{6}) b_{1} \\
-9\left(x_{2}+y_{2}\right) b_{2}-18 b_{1} b_{3} & =(\mu-2 \sqrt{6}) b_{2} \\
-9\left(x_{3}+y_{3}\right) b_{3}-18 b_{1} b_{2} & =(\mu-2 \sqrt{6}) b_{3} \\
-27\left(x_{2} x_{3}\right)-9 b_{1}^{2} & =(\mu-3 \sqrt{6}) x_{1}+\beta \\
-27\left(x_{1} x_{3}\right)-9 b_{2}^{2} & =(\mu-3 \sqrt{6}) x_{2}+\beta \\
-27\left(x_{1} x_{2}\right)-9 b_{3}^{2} & =(\mu-3 \sqrt{6}) x_{3}+\beta
\end{aligned}
$$

and (A10), (A11), and (A12) are obtained by substituting $y_{i}$ in place of $x_{i}(i=1,2,3)$ and $\gamma$ in place of $\beta$ in equations (A7), (A8), (A9).

We now assume $0 \leq b_{1} \leq b_{2} \leq b_{3}, x_{1} \leq x_{2} \leq x_{3}, y_{1} \leq y_{2} \leq y_{3}$, and prove that (A4)-(A12) imply $b_{1}=b_{2}$. To see this, first suppose $b_{1}=0$. Then by (A4), $b_{2} b_{3}=0$; since $b_{2} \leq b_{3}, b_{2}=0$ and thus $b_{1}=b_{2}$.

If $b_{1} \neq 0$, then $b_{2} \neq 0, b_{3} \neq 0$. Subtracting (A8) from (A7) we get

(A13)

$$
-27 x_{3}\left(x_{2}-x_{1}\right)-9\left(b_{1}^{2}-b_{2}^{2}\right)=(\mu-3 \sqrt{6})\left(x_{1}-x_{2}\right) .
$$

Subtracting (A11) from (A10) we get

$$
-27 y_{3}\left(y_{2}-y_{1}\right)-9\left(b_{1}^{2}-b_{2}^{2}\right)=(\mu-3 \sqrt{6})\left(y_{1}-y_{2}\right) .
$$

Adding (A13) and (A14),

(A15)

$$
\begin{aligned}
-18\left(b_{1}^{2}-b_{2}^{2}\right)= & (\mu-3 \sqrt{6})\left(x_{1}+y_{1}-x_{2}-y_{2}\right) \\
& +27\left(x_{3}\left(x_{2}-x_{1}\right)+y_{3}\left(y_{2}-y_{1}\right)\right) .
\end{aligned}
$$


Subtracting (A5) from (A4) and substituting into (A15), we obtain

(A16)

$$
-18\left(b_{1}^{2}-b_{2}^{2}\right)=2(\mu-3 \sqrt{6})\left(-\frac{b_{2} b_{3}}{b_{1}}+\frac{b_{1} b_{3}}{b_{2}}\right)+27 \mathrm{P}
$$

where $\mathrm{P}=x_{3}\left(x_{2}-x_{1}\right)+y_{3}\left(y_{2}-y_{1}\right)$. Observe that $\mathrm{P} \geq 0$.

Thus if $b_{2}^{2}-b_{1}^{2}>0$, we may divide (A16) by $b_{2}^{2}-b_{1}^{2}$ and get

$$
18=-2(\mu-3 \sqrt{6}) \frac{b_{3}}{b_{1} b_{2}}+27 \frac{\mathrm{P}}{b_{2}^{2}-b_{1}^{2}} .
$$

Substituting (A17) into (A6), we get

(A18)

$$
\begin{aligned}
-9\left(x_{3}+y_{3}\right) & =(\mu-3 \sqrt{6})+\sqrt{6}+18 \frac{b_{1} b_{2}}{b_{3}} \\
& =9 \frac{b_{1} b_{2}}{b_{3}}+\frac{27}{2} \frac{\mathrm{P}}{b_{2}^{2}-b_{1}^{2}} \frac{b_{1} b_{2}}{b_{3}}+\sqrt{6} .
\end{aligned}
$$

The left-hand side of (A18) is $\leq 0$, while the right-hand side is $\geq \sqrt{6}>0$. Since this contradicts the hypothesis $b_{2}^{2}-b_{1}^{2}>0$, we must have $b_{2}^{2}=b_{1}^{2}$ and hence $b_{1}=b_{2}$. This establishes Step 2.

Proof of Step 3. - Denote $b_{1}=b_{2}=b$, assume $b=0$, and $b_{3}>0$.

We rewrite (A6), (A13) and (A14) in this case and get

$(\mathbf{A 6})^{\prime}$

$$
\begin{aligned}
-9\left(x_{3}+y_{3}\right) & =(\mu-2 \sqrt{6}), \\
-27 x_{3}\left(x_{2}-x_{1}\right) & =(\mu-3 \sqrt{6})\left(x_{1}-x_{2}\right), \\
-27 y_{3}\left(y_{2}-y_{1}\right) & =(\mu-3 \sqrt{6})\left(y_{1}-y_{2}\right) .
\end{aligned}
$$$$
(\mathbf{A 1 4})^{\prime}
$$

We observe that from (A6)' (and $x_{3} \geq 0, y_{3} \geq 0$ ) that $\mu-2 \sqrt{6} \leq 0$. Thus we conclude from $(\mathrm{A} 13)^{\prime}$ and $(\mathrm{A} 14)^{\prime}$ that $x_{1}=x_{2}$ and $y_{1}=y_{2}$. Denote $x_{1}=x_{2}=-a, y_{1}=y_{2}=-c$, with $a, c \geq 0$. Then $\overrightarrow{\mathrm{B}}=\left(0,0, b_{3}\right), \overrightarrow{\mathrm{X}}=(-a,-a, 2 a), \overrightarrow{\mathrm{Y}}=(-c,-c, 2 c)$. We now assert that $a=c$. To see this, we first subtract (A9) from (A8) to get

(A19)

$$
-27 x_{1}\left(x_{3}-x_{2}\right)+9 b_{3}^{2}=(\mu-3 \sqrt{6})\left(x_{2}-x_{3}\right)
$$

or

$(\mathbf{A 1 9})^{\prime}$

$$
27 a^{2}+3 b_{3}^{2}=-(\mu-3 \sqrt{6}) a .
$$

Similarly we subtract (A12) from (A11) and get

$$
27 c^{2}+3 b_{3}^{2}=-(\mu-3 \sqrt{6}) c .
$$


Subtracting (A20)' from (A19)', we then have

$$
27\left(a^{2}-c^{2}\right)=-(\mu-3 \sqrt{6})(a-c)
$$

Thus, either $a=c$, or $a \neq c$ while

$$
-27(a+c)=\mu-3 \sqrt{6} .
$$

We will now derive a contradiction to see that the latter possibility $(a \neq c)$ does not happen. Comparing (A21) with $(\mathrm{A} 6)^{\prime}$, we see $-18(a+c)=\mu-2 \sqrt{6}$. Combining (A21) with $(\mathrm{A} 6)^{\prime}$ we get

(A22)

$$
a+c=\frac{\sqrt{6}}{9} .
$$

We now add (A19) to (A20) and substitute the relation $\varphi_{1}=2 b_{3}^{2}+6\left(a^{2}+c^{2}\right)=1$ and (A21) into the equation to get

(A23)

$$
3\left(a^{2}+c^{2}\right)=9(a+c)^{2}-1
$$

By (A22), this expression equals $\frac{6}{9}-1<0$, which is a contradiction. Thus we conclude that $a=c$ and $\overrightarrow{\mathrm{B}}=\left(0,0, b_{3}\right), \overrightarrow{\mathrm{X}}=\overrightarrow{\mathrm{Y}}=(-a,-a, 2 a)$ for some $b_{3}>0$ and $a \geq 0$. At this moment, we can check directly that $\mathrm{I}=\mathrm{I}(\overrightarrow{\mathrm{B}}, \overrightarrow{\mathrm{X}}, \overrightarrow{\mathrm{Y}})>0$ if $b_{3}>0$. To be more precise, one can check that $\mathrm{I}=8\left(\mathrm{I}_{1}-\mathrm{I}_{2}\right)$ with $\mathrm{I}_{1}=\sqrt{3}\left(b_{3}^{2}+9 a^{2}\right)\left(b_{3}^{2}+6 a^{2}\right)^{1 / 2}$, $\mathrm{I}_{2}=9 a\left(3 a^{2}+b_{3}^{2}\right)$, and $\mathrm{I}_{1}^{2}-\mathrm{I}_{2}^{2}>0$. This establishes Step 3 .

Proof of Step 4. - Assume $b_{1}=b_{2}=b \neq 0, b_{3}>0$. In this case, we may write (A4), (A5), (A6) as

$$
\begin{array}{ll}
(\mathbf{A 4})^{\prime \prime} & -9\left(x_{1}+y_{1}\right)-18 b_{3}=\mu-2 \sqrt{6}, \\
(\mathbf{A 5})^{\prime \prime} & -9\left(x_{2}+y_{2}\right)-18 b_{3}=\mu-2 \sqrt{6}, \\
(\mathbf{A 6})^{\prime \prime} & -9\left(x_{3}+y_{2}\right) b_{3}-18 b^{2}=(\mu-2 \sqrt{6}) b_{3} .
\end{array}
$$

Subtracting (A5)" from (A4)", we get

$(\mathbf{A} 24)$

$$
x_{1}+y_{1}=x_{2}+y_{2} .
$$

Subtracting (A8) from (A7) and (A11) from (A10) we get

$$
\begin{array}{ll}
(\mathbf{A 1 3})^{\prime \prime} & -27 x_{3}\left(x_{2}-x_{1}\right)=(\mu-3 \sqrt{6})\left(x_{1}-x_{2}\right), \\
(\mathbf{A 1 4})^{\prime \prime} & -27 y_{3}\left(y_{2}-y_{1}\right)=(\mu-3 \sqrt{6})\left(y_{1}-y_{2}\right) .
\end{array}
$$


We now make two claims.

Glaim 1: $x_{1}=x_{2}(=-a), y_{1}=y_{2}(=-c)$.

Claim 2: $a=c$.

Proof of Claim 1. - By (A13)", we have either $x_{1}=x_{2}$ or $x_{1} \neq x_{2}$ and $27 x_{3}=$ $\mu-3 \sqrt{6}$. By $(\mathrm{A} 14)^{\prime \prime}$, we have either $y_{1}=y_{2}$ or $y_{1} \neq y_{2}$ and $27 y_{3}=\mu-3 \sqrt{6}$. By (A24), we have $x_{1}=x_{2}$ implies $y_{1}=y_{2}$. Thus, we either have $x_{1}=x_{2}$ and $y_{1}=y_{2}$ as claimed, or $x_{1} \neq x_{2}, y_{1} \neq y_{2}$ while $x_{3}=y_{3}=\frac{1}{27}(\mu-3 \sqrt{6})$. But in the latter case, from(A6)" we would have

$$
-9\left(x_{3}+y_{3}\right) b_{3}-18 b^{2}=(\mu-2 \sqrt{6}) b_{3}=\left(27 x_{3}+\sqrt{6}\right) b_{3} .
$$

This is a contradiction as the left-hand side of the equation is less than zero, while the right-hand side is bigger than zero.

Proof of Claim 2. - We follow the same strategy as in the proof of Step 3. Subtracting (A9) from (A8) we get

$$
(\mathbf{A 1 9})^{\prime \prime} \quad 27 a^{2}+3\left(b_{3}^{2}-b^{2}\right)=-a(\mu-3 \sqrt{6}) .
$$

Similarly, if we subtract (A12) from (A11) we get

$$
(\mathbf{A 2 0})^{\prime \prime} \quad 27 c^{2}+3\left(b_{3}^{2}-b^{2}\right)=-c(\mu-3 \sqrt{6}) .
$$

Finally, subtracting (A20)" from (A19)" we have

$$
27\left(a^{2}-c^{2}\right)=-(\mu-3 \sqrt{6})(a-c) .
$$

Thus, either $a=c$ as claimed, or

$$
(\mathbf{A 2 1})^{\prime \prime} \quad a \neq c \text { and } 27(a+c)=-(\mu-3 \sqrt{6}) \text {. }
$$

We will now show that $(\mathrm{A} 21)^{\prime \prime}$ cannot be true. To see this, denote $a+c=\ell(\ell \geq 0)$, $a-c=d$. Then $d \neq 0$, and w.l.o.g. we may assume $d>0$. Substituting (A21)" into (A4) we get

$(\mathbf{A 2 4})$

$$
\begin{aligned}
-18 b_{3} & =(\mu-2 \sqrt{6})-9(a+c) \\
& =(\mu-3 \sqrt{6})-9 \ell+\sqrt{6}=-36 \ell+\sqrt{6} .
\end{aligned}
$$

Substituting (A21)" to (A6) we get

(A25)

$$
\begin{aligned}
-18 b^{2} & =(\mu-2 \sqrt{6}) b_{3}+18(a+c) b_{3} \\
& =(-9 \ell+\sqrt{6}) b_{3} .
\end{aligned}
$$

Thus in particular

$(\mathbf{A 2 5})^{\prime \prime} \quad-9 \ell+\sqrt{6}<0$. 
Combining (A24) and (A25), we get

(A26)

$$
18\left(b^{2}-b_{3}^{2}\right)=(-36 \ell+\sqrt{6}+9 \ell-\sqrt{6}) b_{3}=-27 \ell b_{3} .
$$

On the other hand, substituting (A21)" into (A19)", we get

$$
27 a^{2}+3\left(b_{3}-b^{2}\right)=27 a(a+c),
$$

So

$(\mathbf{A} 27)$

$$
3\left(b_{3}^{2}-b^{2}\right)=27 a c=27 \frac{\ell^{2}-d^{2}}{4} .
$$

Combining (A24), (A26), (A27), we get

(A28)

$$
27 d^{2}=(-9 \ell+\sqrt{6}) \ell
$$

which contradicts (A25)". We conclude $a=c$, as in Claim 2.

We are now in the situation where $\overrightarrow{\mathrm{B}}=\left(b, b, b_{3}\right)$, with $b_{3} \geq b>0$, and $\overrightarrow{\mathrm{X}}=$ $\overrightarrow{\mathrm{Y}}=(-a,-a, 2 a)$. There are two final possibilities to consider, depending on the sign of $a$.

Claim 3: If $a=0$, then $b_{3}=b \neq 0$, and $\mathrm{I} \equiv 0$.

Glaim 4: If $a>0$ then I $>0$.

Proof of Claim 3. - When $a=0$, we multiply (A4)" by $b_{3}$ then subtract (A6)" to get $b_{3}^{2}=b^{2}$, hence $b_{3}=b$. In this case $\mathrm{I}=72 b^{3}-72 b^{3} \equiv 0$.

Proof of Claim 4. - When $a \neq 0$, we will show that $\mathrm{I}>0$. First, we rewrite $(\mathrm{A} 4)^{\prime \prime},(\mathrm{A} 6)^{\prime \prime},(\mathrm{A} 7)$ and (A9) as follows:

$$
\begin{array}{lrl}
(\mathbf{A 4})^{\prime \prime} & 18 a-18 b_{3} & =\mu-2 \sqrt{6}, \\
(\mathbf{A 6})^{\prime \prime} & -36 a b_{3}-18 b^{2} & =(\mu-2 \sqrt{6}) b_{3}, \\
(\mathbf{A 7})^{\prime \prime} & 54 a^{2}-9 b^{2} & =-(\mu-3 \sqrt{6}) a+\beta, \\
(\mathbf{A 9})^{\prime \prime} & -27 a^{2}-9 b_{3}^{2}=2(\mu-3 \sqrt{6}) a+\beta .
\end{array}
$$

Multiplying (A4)" by $b_{3}$ and subtracting (A6)" from the result we get

(A29) $\quad b_{3}^{2}-b^{2}=3 a b_{3}$.

Subtracting (A9)" from (A7)" we get

(A30)

$$
81 a^{2}+9\left(b_{3}^{2}-b^{2}\right)=-3 a(\mu-3 \sqrt{6}) .
$$

Combining (A29) and (A30), we get (for $a \neq 0$ )

(A31)

$$
27 a+9 b_{3}=-(\mu-3 \sqrt{6}) .
$$


Combining (A4)" and (A31) we find

(A32)

$$
45 a-9 b_{3}=\sqrt{6} .
$$

Substituting (A29) into the constraint $\varphi_{1}=2\left(2 b^{2}+b_{3}^{2}\right)+12 a^{2}=1$ we get

$(\mathbf{A 3 3})$

$$
6 b_{3}^{2}-12 a b_{3}+12 a^{2}=1 .
$$

We now introduce the notation $s=a-b_{3}$ and rewrite (A32) and (A33) as

(A32)

$$
\begin{gathered}
36 a+9 s=\sqrt{6}, \\
6\left(s^{2}+a^{2}\right)=1 .
\end{gathered}
$$

$(\mathbf{A 3 3})^{\prime}$

Applying (A29) we can write I as

$$
\begin{aligned}
\mathrm{I} & =\left(4\left(2 b^{2}+b_{3}^{2}\right)+36 a^{2}\right) \sqrt{6}-216 a^{3}-72 b^{2} b_{3}-72 a\left(b_{3}^{2}-b^{2}\right) \\
& =12 \sqrt{6}\left(b_{3}^{2}-2 a b_{3}+3 a^{2}\right)-72\left(3 a^{3}+b_{3}^{2}-3 a^{2} b_{3}+3 a b_{3}^{2}\right) \\
& =72\left[\frac{s^{2}+2 a^{2}}{\sqrt{6}}-\left(4 a^{3}-s^{3}\right)\right] .
\end{aligned}
$$

It remains to check numerically that a solution $(a, s)$ with $a>0$ of equations $(\mathrm{A} 32)^{\prime}$ and $(\mathrm{A} 33)^{\prime}$ is given by $a=0.1617 \ldots, s=-0.3746 \ldots$, and $\mathrm{I}=72(0.079 \ldots$ $-0.069 \ldots)>0$. tion A.

We have thus finished the proof of Step 4 and completed the proof of Proposi-

\section{REFERENGES}

[Ad] D. Adams, A sharp inequlity of J. Moser for higher derivatives. Annals of Math., 128 (1988), 385-398.

[Be] A. Besse, Einstein Manifolds. Berlin: Springer-Verlag (1987).

[BO] T. Branson and B. Orsted, Explicit functional determinants in four dimensions. Proc. A.M.S., 113 (1991), 669-682.

[GGY1] S. Y. A. Chang, M. J. Gursky, and P. Yang, An equation of Monge-Ampere type in conformal geometry, and four-manifolds of positive Ricci curvature. Annals of Math., 155 (2002), 711-789.

[CGY2] S. Y. A. Chang, M. J. Gursky, and P. Yang, An a priori estimate for a fully nonlinear equation on fourmanifolds. F. D'Analyse Math., 87 (2002), to appear.

[CGY3] S. Y. A. Chang, M. J. Gursky, and P. YAng, Regularity of a fourth order nonlinear PDE with critical exponent. Amer. 7. Math., 121 (1999), 215-257.

[CYl] S. Y. A. Chang and P. Yang, Extremal metrics of zeta function determinants on 4-manifolds. Annals of Math., 142 (1995), 171-212.

[CMS] J. Cheeger, W. Müller, and R. Schrader, On the curvature of piecewise flat spaces. Comm. Math. Phys., 92 (1984), 405-454.

[De] A. Derdzinski, Self-dual Kähler manifolds and Einstein manifolds of dimension four. Compositio Math., 49 (1983), 405-433.

[Ev] L. C. Evans, Classical solutions of fully nonlinear, convex, second-order elliptic equations. Comm. Pure Appl. Math., 35 (1982), 333-363.

[Fr] M. Freedman, The topology of four-dimensional manifolds. F. Diff. Geom., 17 (1982), 357-453. 
[Gu] M. Gursky, The Weyl functional, deRham cohomology, and Kähler-Einstein metrics. Annals of Math., 148 (1998), 315-337.

[GT] D. Gilbarg and N. Trudinger, Elliptic Partial Differential Equations of Second Order. Berlin, Heidelberg: Springer (1983).

[Ha] R. Hamilton, Four-manifolds with positive curvature operator. F. Diff. Geom., 24 (1986), 153-179.

[Hi] N. Hitchin, On compact four-dimensional Einstein manifolds. F. Diff. Geom., 9 (1974), 435-442.

[Hu] G. Huisken, Ricci deformation of the metric on a Riemannian manifold. J. Diff. Geom., 21 (1985), 47-62.

[Kr] N. V. KrYLov, Boundedly inhomogeneous elliptic and parabolic equations in a domain. Izv. Akad. Mauk. SSSR Ser. Mat., 47 (1983), 75-108.

[L] Y. LI, Degree theory for second order nonlinear elliptic operators and its applications. Comm. PDE, 14 (1989), 1547-1578.

[Mal] G. Margerin, Pointwise pinched manifolds are Spaceforms 44 (1986). AMS Proc. of Symp. in Pure Math., Arcata '84, 307-328.

[Ma2] G. Margerin, A sharp characterization of the smooth 4-sphere in curvature terms. Comm. Anal. Geom., 6 (1998), 21-65.

[Pe] P. Petersen, Riemannian Geometry. Springer Graduate Texts in Mathematics 171. New York (1998).

[ST] I. Singer and J. ThORPe, The curvature of four-dimensional Einstein spaces, in Global Analysis (Papers in honor of K. Kodaira), D. Spencer and S. Iyanaga (eds.), pp. 355-365. Tokyo: University of Tokyo Press (1969).

[UV] K. Uhlenbeck and J. Viaclovsky, Regularity of weak solutions to critical exponent variational equations. Math. Res. Lett., 7 (2000), 651-656.

S.-Y.A. C.

University of Princeton

Department of Mathematics

Washington Road, Fine Hall

Princeton, NJ 08544-1000, USA

chang@math.princeton.edu

M.J. G.

University of Notre Dame

Department of Mathematics

Notre Dame, IN 46556-5670, USA

mgursky@nd.edu

P.C. Y.

University of Princeton

Department of Mathematics

Washington Road, Fine Hall

Princeton, NJ 08544-1000, USA

yang@math.princeton.edu

Manuscrit reçu le 13 juillet 2002. 